\title{
Faster 3-Periodic Merging Networks
}

\author{
Marek Piotrów \\ Institute of Computer Science, University of Wrocław, \\ ul. Joliot-Curie 15, PL-50-383 Wrocław, Poland \\ Marek.Piotroweii.uni.wroc.pl
}

\begin{abstract}
We consider the problem of merging two sorted sequences on a comparator network that is used repeatedly, that is, if the output is not sorted, the network is applied again using the output as input. The challenging task is to construct such networks of small depth. The first constructions of merging networks with a constant period were given by Kutyłowski, Loryś and Oesterdikhoff [7]. They have given 3-periodic network that merges two sorted sequences of $N$ numbers in time $12 \log N$ and a similar network of period 4 that works in $5.67 \log N$. We present a new family of such networks that are based on Canfield and Williamson periodic sorter [4]. Our 3-periodic merging networks work in time upper-bounded by $6 \log N$. The construction can be easily generalized to larger constant periods with decreasing running time, for example, to 4-periodic ones that work in time upper-bounded by $4 \log N$. Moreover, to obtain the facts we have introduced a new proof technique.
\end{abstract}

Keywords: parallel merging, comparison networks, merging networks, periodic networks, comparators, oblivious merging.

\section{Introduction}

Comparator networks are probably the simplest parallel model that is used to solve such tasks as sorting, merging or selecting [6]. Each network represents a data-oblivious algorithm, which can be easily implemented in hardware. Moreover, sorting networks can be applied in secure, multi-party computation (SMC) protocols. They are also strongly connected with switching networks [9]. The most famous constructions of sorting networks are Odd-Even and Bitonic networks of depth $\frac{1}{2} \log ^{2} N$ due to Batcher [2] and AKS networks of depth $O(\log N)$ due to Ajtai, Komlos and Szemeredi [1]. The longstanding disability to decrease a large constant hidden behind the asymptotically optimal complexity of AKS networks to a practical value [15] has resulted in studying easier, sorting-related problems, whose optimal networks have small constants.

A comparator network consists of a set of $N$ registers, each of which can contain an item from a totally ordered set, and a sequence of comparator stages. Each stage is a set of comparators that connect disjoint pairs of registers and, therefore, can work in parallel (a comparator is a simple device that takes a contents of two registers and performs a compare-exchange operation on them: the minimum is put into the first register and the maximum into the second one). Stages are run one after another in 
synchronous manner, hence we can consider the number of stages as the running time. The size of a network is defined to be the total number of comparators in all its stages.

A network $A$ consisting of stages $S_{1}, S_{2}, \ldots, S_{d}$ is called $p$-periodic if $p<d$ and for each $i, 1 \leq i \leq d-p$, stages $S_{i}$ and $S_{i+p}$ are identical. A periodic network is easy to implement, especially in hardware, because one can use the first $p$ stages in a cycle: if the output of $p$-th stage is not correct (sorted, for example), the sequence of $p$ stages is run again. We can also define a $p$-periodic network just by giving the total number of stages and a description of its first $p$ stages. A challenging task is to construct a family of small-periodic networks for sorting-related problems with the running time equal to, or not much greater than that of non-periodic networks.

Dowd et al. [5] gave the construction of $\log N$-periodic sorting networks of $N$ registers with running time of $\log ^{2} N$. Kutyłowski et al. [8] introduced a general method to convert a non-periodic sorting network into a 5-periodic one, but the running time increases by a factor of $O(\log N)$ during the conversion. For simpler problems such as merging or correction there are constant-periodic networks that solve the corresponding problem in asymptotically optimal logarithmic time [7]13]. In particular, Kutyłowski, Loryś and Oesterdikhoff [7] have given 3-periodic network that merges two sorted sequences of $N$ numbers in time $12 \log N$ and a similar network of period 4 that works in $5.67 \log N$. They have also sketched a construction of merging networks with periods larger than 4 and running time decreasing asymptotically to $2.25 \log N$.

In this paper, we introduce a new family of constant-periodic merging networks that are based on the Canfield and Williamson $O(\log N)$-periodic sorter [4] by a certain periodification technique. Our 3-periodic merging networks work in time upper-bounded by $6 \log N$ and 4 -periodic ones - in time upper-bounded by $4 \log N$. The construction can be easily generalized to larger constant periods with decreasing running time.

The advantage of constant-periodic networks is that they have pretty simple patterns of communication links, that is, each node (register) of such a network can only be connected to a constant number of other nodes. Such patterns are easier to implement, for example, in hardware. Moreover, a node uses these links in a simple periodic manner and this can save control login and simplify timing considerations.

\section{Periodic merging networks}

Our merging networks are based on the Canfield and Williamson [4] $O(\log N)$-periodic sorters. We recall now the definition of their networks: for each $k \geq 1$ let $C W_{k}=$ $S_{1}, \ldots, S_{k}$ denote a network of $N=2^{k}$ registers, where the stages are defined as follows (see also Figures 1 and 2):

$$
\begin{aligned}
S_{1} & =\left\{[2 i: 2 i+1]: i=0,1, \ldots, 2^{k-1}-1\right\}, \\
S_{j+1} & =\left\{\left[2 i+1: 2 i+2^{k-j}\right]: i=0,1, \ldots, 2^{k-1}-2^{k-j-1}-1\right\}, j=1, \ldots, k-1 .
\end{aligned}
$$

The merging and sorting properties of the networks are given in the following proposition. 


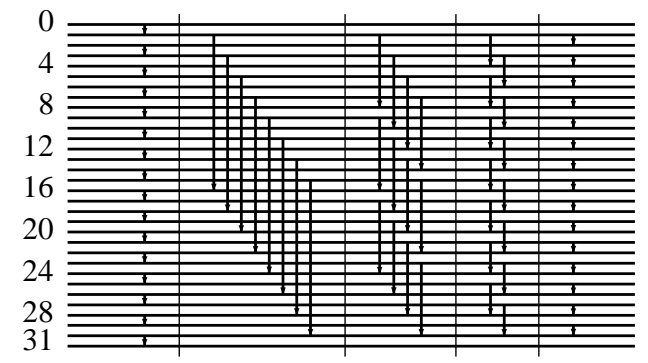

Fig. 1. The Canfield and Williamson $\log N$-periodic sorter, where $N=32$. Registers and comparators are represented by horizontal lines and arrows, respectively. Stages are separated by vertical lines.

Proposition 1. (1) For each $k \geq 1$, if two sorted sequences of length $2^{k-1}$ are given in registers with odd and even indices, respectively, then $C W_{k}$ is a merging network. (2) For each $k \geq 1, C W_{k}$ is a $k$-pass periodic sorting network.

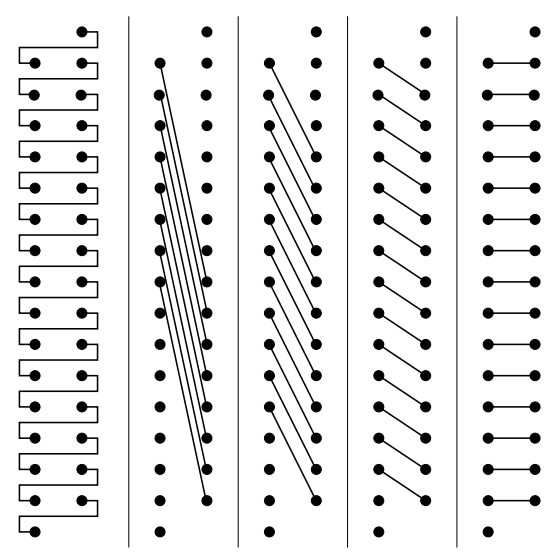

Fig. 2. Another view of $C W_{5}$ 5-pass 5-periodic sorter. Registers and comparators are represented by dots and edges, respectively. Stages are separated by vertical lines.

We would like to implement a version of this network as a constant-periodic comparator network. Consider first the most challenging 3-periodic implementation. We start with the definition of a temporally construction $P_{k}$ which structure is similar to the structure of $C W_{k}$. Then we transform it to 3-periodic network $M_{k}$. The idea is to replace each register $i$ in $C W_{k}$ (except the first and the last ones) with a sequence of $k-2$ consecutive registers, move the endpoints of long comparators one register further or closer depending on the parity of $i$ and insert between each pair of stages containing long com- 
parators a stage with short comparators joining the endpoints of those long ones. The result is depicted in Fig. 3. In this way, we obtain a network in which each register is used in at most three consecutive stages. Therefore the network $P_{k}$ can be packed into the first 3 stages and used periodically to get the desired 3-periodic merging network.

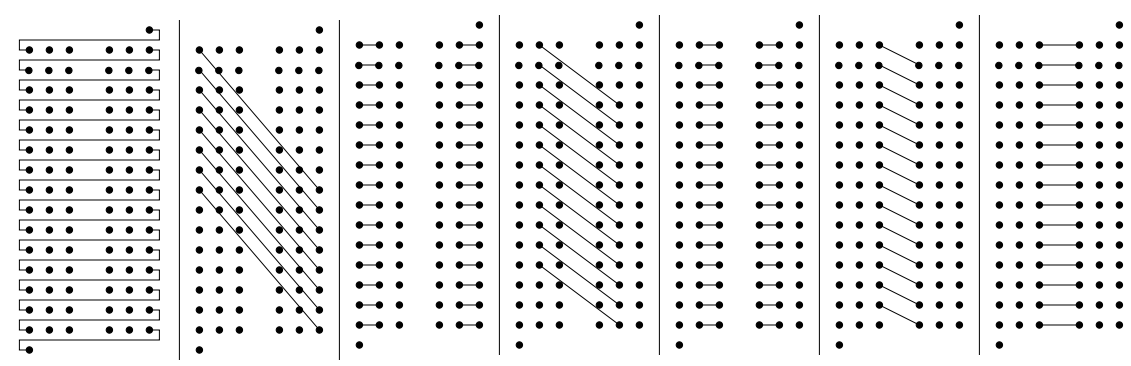

Fig. 3. $P_{5}$ as an implementation of $C W_{5}$. Registers and comparators are represented by dots and edges, respectively. Stages are separated by vertical lines. Stages with short horizontal comparators are inserted between stages with long comparators.

Let $[i: j]$ denote a comparator connecting registers $i$ and $j$. A comparator $[i: j]$ is standard if $i<j$. For an $N$-register network $A=S_{1}, S_{2}, \ldots, S_{d}$, where $S_{1}, S_{2}, \ldots, S_{d}$ denote stages, and for an integer $j \in\{1, \ldots, N\}$, we will use the following notations:

$$
\begin{aligned}
f s t(j, A) & =\min \left\{1 \leq i \leq d: j \in \operatorname{regs}\left(S_{i}\right)\right\} \\
\operatorname{lst}(j, A) & =\max \left\{1 \leq i \leq d: j \in \operatorname{regs}\left(S_{i}\right)\right\} \\
\operatorname{delay}(A) & =\max _{j \in\{1, \ldots, N\}}\{l s t(j, A)-f s t(j, A)+1\}
\end{aligned}
$$

where $\operatorname{regs}\left(\left\{\left[i_{1}: j_{1}\right], \ldots,\left[i_{r}: j_{r}\right]\right\}\right)$ denotes the set $\left\{i_{1}, j_{1}, \ldots, i_{r}, j_{r}\right\}$.

Let us define formally the new family of merging networks. For each $k \geq 3$ we would like to transform the network $C W_{k}$ into a new network $P_{k}$.

Definition 1. Let $n_{k}=2^{k-1}-1$ be one less than the half of the number of registers in $C W_{k}$ and $b_{k}=2(k-2)$. The number of registers of $P_{k}$ is defined to be $N_{k}=n_{k} \cdot b_{k}+2$. The stages of $P_{k}=S_{k, 1} \cup\left\{[0: 1],\left[N_{k}-2: N_{k}-1\right]\right\}, S_{k, 2}, \ldots, S_{k, 2 k-3}$ are defined by the following equations, where $j=1, \ldots, \frac{b_{k}}{2}$ :

$$
\begin{aligned}
S_{k, 1}= & \left\{\left[b_{k} i: b_{k} i+1\right]: i=1, \ldots, n_{k}-1\right\} \\
S_{k, 2 j}= & \left\{\left[b_{k} i+j: b_{k}\left(i+2^{k-j-1}-1\right)+\left(b_{k}-j+1\right)\right]: i=0, \ldots, n_{k}-2^{k-j-1}\right\} \\
S_{k, 2 j+1}= & \left\{\left[b_{k} i+j: b_{k} i+j+1\right],\right. \\
& {\left.\left[b_{k} i+\left(b_{k}-j\right): b_{k} i+\left(b_{k}-j+1\right)\right]: i=0, \ldots, n_{k}-1\right\} }
\end{aligned}
$$

The network $P_{5}$ is depicted in Figure 4.

Fact 1 delay $\left(P_{k}\right)=3$ for $k \geq 3$. 


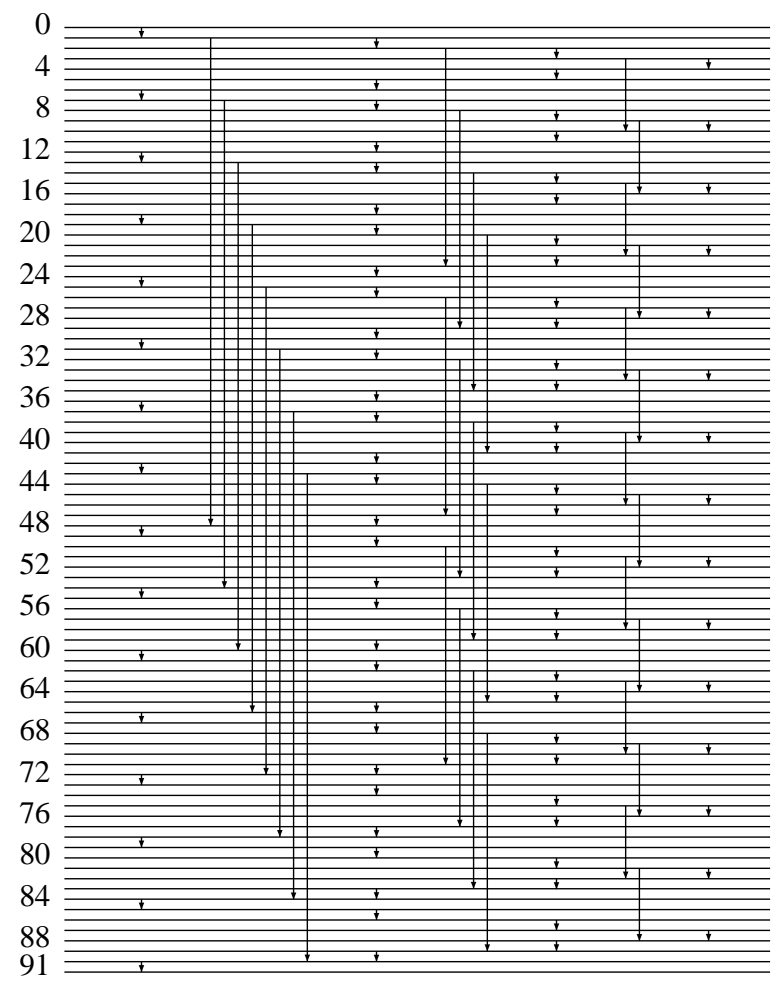

Fig. 4. The traditional drawing of $P_{5}$ network

Let $A=S_{1}, S_{2}, \ldots, S_{d}$ and $A^{\prime}=S_{1}^{\prime}, S_{2}^{\prime}, \ldots, S_{d^{\prime}}^{\prime}$ be $N$-input comparator networks such that for each $i, 1 \leq i \leq \min \left(d, d^{\prime}\right), \operatorname{regs}\left(S_{i}\right) \cap \operatorname{regs}\left(S_{i}^{\prime}\right)=\emptyset$. Then $A \cup A^{\prime}$ is defined to be $\left(S_{1} \cup S_{1}^{\prime}\right),\left(S_{2} \cup S_{2}^{\prime}\right), \ldots,\left(S_{\max \left(d, d^{\prime}\right)} \cup S_{\max \left(d, d^{\prime}\right)}^{\prime}\right)$, where empty stages are added at the end of the network of smaller depth.

For any comparator network $A=S_{1}, \ldots, S_{d}$ and $D=\operatorname{delay}(A)$, let us define a network $B=T_{1}, \ldots, T_{D}$ to be a compactform of $A$, where $T_{q}=\bigcup\left\{S_{q+p D}: 0 \leq p \leq(d-q) / D\right\}$, $1 \leq q \leq D$. Observe that $B$ is correctly defined due to the delay of $A$. Moreover, $\operatorname{depth}(B)=$ $\operatorname{delay}(B)=\operatorname{delay}(A)$.

Definition 2. For $k \geq 3$ let $M_{k}$ denote the compact form of $P_{k}$ with the first and the last registers deleted. That is, the network $M_{k}=T_{1}^{k}, T_{2}^{k}, T_{3}^{k}$ is using the set of registers numbered $\left\{1,2, \ldots, N_{k}\right\}$, where $N_{k}=\left(2^{k-1}-1\right) \cdot 2(k-2)$, and $T_{j}^{k}=\left\{S_{k, j+3 i}: 0 \leq i \leq\right.$ $\left.\frac{2 k-j-3}{3}\right\}, j=1,2,3$.

It is not necessary to delete the first and the last registers of $P_{k}$ but this will simplify proofs a little bit in the next section. The network $M_{5}$ is given in Fig.5.

Theorem 2. There exists a family of 3-periodic comparator networks $M_{k}, k \geq 3$, such that each $M_{k}$ is a $2 k-5$-pass merger of two sorted sequences given in odd and even 


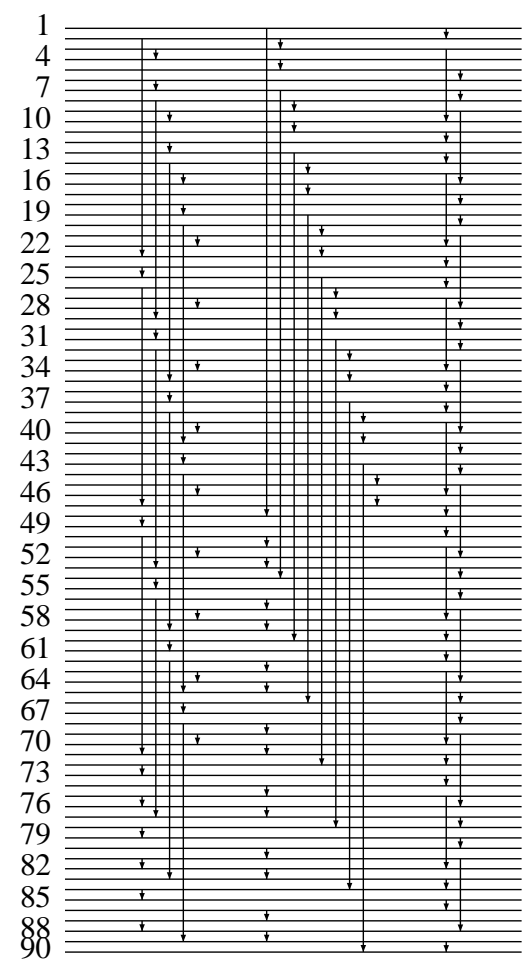

Fig. 5. The $M_{5}$ network

registers, respectively. The running time of $M_{k}$ is $6 k-15 \leq 6 \log N_{k}$, where $N_{k}=\left(2^{k}-\right.$ $2)(k-2)$ is the number of registers in $M_{k}$.

The proof is based on the observation that $M_{k}$ merges $k-2$ pairs of sorted subsequences, one after another, in pipeline fashion. Details are given in the next section.

In a similar way, we can convert $C W_{k}$ into a 4-periodic merging network. Assume that $k$ is even. We replace each register (except the first and the last ones) with a sequence of $(k-2) / 2$ consecutive registers, move the endpoints of long comparators in such a way that exactly two long comparators start or end at each new register and insert after each pair of stages containing long comparators a stage with short comparators joining the endpoints of those long comparators. The result is depicted in Fig. 6 .

\section{Proof of Theorem 2}

The first observation we would like to make is that we can consider inputs consisting of 0's and 1's only. The well-known Zero-One Principle states that any comparator network that sorts $0-1$ input sequences correctly sorts also arbitrary input sequences [6]. In the similar way, we can prove that the same property holds also for merging: 
Proposition 2. If a comparator network merges any two 0-1 sorted sequences, then it correctly merges any two sorted sequences.

Therefore we can analyze computations of the network $M_{k}, k \geq 3$, by describing each state of registers as a $0-1$ sequence $\bar{x}=\left(x_{1}, \ldots, x_{N_{k}}\right)$, where $x_{i}$ represents the content of register $i$. If $\bar{x}$ is an input sequence for $2 k-5$ passes of $M_{k}$, then by $\bar{x}^{(i)}$ we denote the content of registers after $i$ passes of $M_{k}, i=0, \ldots, 2 k-5$, that is, $\bar{x}^{(0)}=\bar{x}$ and $\bar{x}^{(i+1)}=$ $M_{k}\left(\bar{x}^{(i)}\right)$. Since $M_{k}$ consists of three stages $T_{1}^{k}, T_{2}^{k}$ and $T_{3}^{k}$, we extend the notation to describe the output of each stage: $\bar{x}^{(i, 0)}=\bar{x}^{(i)}$ and $\bar{x}^{(i, j)}=T_{j}^{k}\left(\bar{x}^{(i, j-1)}\right)$, for $j=1,2,3$. For other values of $j$ we assume that $\bar{x}^{(i, j)}=\bar{x}^{(i+j \operatorname{div} 3, j \bmod 3)}$. We will use this superscript notation for other equivalent representations of sequence $\bar{x}$.

Now let us fix some technical notations and definitions. A 0-1 sequence can be represented as a word over $\Sigma=\{0,1\}$. A non-decreasing (also called sorted) $0-1$ sequence has a form of $0^{*} 1^{*}$ and can be equivalently represented by the number of ones (or zeros) in it. For any $x \in \Sigma^{*}$ let ones $(x)$ denote the number of 1 in $x$. If $x \in \Sigma^{n}$ then $x_{i}$, $1 \leq i \leq n$, denotes the $i$-th letter of $x$ and $x_{A}, A=\left\{i_{1}, \ldots, i_{m}\right\}, 1 \leq i_{1}<\ldots,<i_{m} \leq n$ denotes the word $x_{i_{1}} \ldots x_{i_{m}}$. We say that a $0-1$ sequence $\bar{x}=\left(x_{1}, \ldots, x_{N_{k}}\right)$ is 2-sorted if both $\left(x_{1}, x_{3}, \ldots, x_{N_{k}-1}\right)$ and $\left(x_{2}, x_{4}, \ldots, x_{N_{k}}\right)$ are sorted.

\subsection{Reduction to Analysis of Columns}

For any $k \geq 3$ let $n_{k}=2^{k-1}-1, b_{k}=2(k-2)$ (thus $N_{k}=n_{k} \cdot b_{k}$ ). The set of registers $\operatorname{Reg}_{k}=\left\{1, \ldots, N_{k}\right\}$ can be analyzed as an $n_{k} \times b_{k}$ matrix with $C_{j}^{k}=\left\{j+i b_{k}: 0 \leq i<n_{k}\right\}$, $j=1, \ldots, b_{k}$, as columns. A content of all registers in the matrix, that is $x \in \Sigma^{N_{k}}$, can be equivalently represented by the sequence of contents of registers in $C_{1}, C_{2}, \ldots, C_{b_{k}}$, that is $\left(x_{C_{1}}, \ldots, x_{C_{b_{k}}}\right)$. Since $b_{k}$ is an even number, the following fact is obviously true.

Fact 3 If $x \in \Sigma^{N_{k}}$ is 2-sorted then each $x_{C_{j}}, j=1, \ldots, b_{k}$, is sorted.

That is, the columns are sorted at the beginning of a computation of $2 k-5$ passes of $M_{k}$. The first lemma we would like to prove is that columns remain sorted after each stage of the computation. We start with a following technical fact:

Fact 4 Let $A=\left\{a_{1}, \ldots, a_{n}\right\}$ and $B=\left\{b_{1}, \ldots, b_{n}\right\}$ be subsets of $\left\{1, \ldots, N_{k}\right\}$ such that $a_{1}<b_{1}<a_{2}<b_{2}<\ldots<a_{n}<b_{n}$. Let $h \geq 0$ and $S_{A, B, h}=\left\{\left[a_{i}: b_{i+h}\right]: 1 \leq i \leq n-h\right\}$. Then for any $x \in \Sigma^{N_{k}}$ such that $x_{A}$ and $x_{B}$ are sorted, the output $y=S_{A, B, h}(x)$ has the following properties:

(i) $y_{A}$ and $y_{B}$ are sorted.

(ii) Let $m_{1}=$ ones $\left(x_{A}\right)$ and $m_{2}=$ ones $\left(x_{B}\right)$. Then ones $\left(y_{A}\right)=\min \left(m_{1}, m_{2}+h\right)$ and ones $\left(y_{B}\right)=\max \left(m_{1}-h, m_{2}\right)$.

Proof. To prove (i) we show only that $y_{a_{i}} \leq y_{a_{i+1}}$ for $i=1, \ldots, n-1$. If $1 \leq i<n-h$ then $y_{a_{i}}=\min \left(x_{a_{i}}, x_{b_{i+h}}\right) \leq \min \left(x_{a_{i+1}}, x_{b_{i+h+1}}\right)=y_{a_{i+1}}$ since min is a non-decreasing function and both $x_{A}$ and $x_{B}$ are sorted. If $i=n-h$ then $y_{a_{i}}=\min \left(x_{a_{i}}, x_{b_{i+h}}\right) \leq x_{a_{i+1}}=y_{a_{i+1}}$. For $i>n-h$ we have $y_{a_{i}}=x_{a_{i}} \leq x_{a_{i+1}}=y_{a_{i+1}}$. 
To prove (ii) let $m_{1}^{\prime}=\min \left(m_{1}, m_{2}+h\right)$ and $m_{2}^{\prime}=\max \left(m_{1}-h, m_{2}\right)$. We consider two cases. If $m_{1} \leq m_{2}+h$ then $m_{1}-h \leq m_{2}$ and we get $m_{1}^{\prime}=m_{1}$ and $m_{2}^{\prime}=m_{2}$. In this case no comparator from $S_{A, B, h}$ exchanges 0 with 1 . To see this assume a.c. that a comparator $\left[a_{i}: b_{i+h}\right]$ exchanges $x_{a_{i}}=1$ with $x_{b_{i+h}}=0$. Then $i>n-m_{1}$ and $i+h \leq n-m_{2}$ hold because of the definitions of $m_{1}$ and $m_{2}$. It follows that $n-m_{1}<n-m_{2}-h$, thus $m_{1}-h>m_{2}-$ a contradiction. If $m_{1}>m_{2}+h$ then $m_{1}^{\prime}=m_{2}+h$ and $m_{2}^{\prime}=m_{1}-h$. In this case let us observe that a comparator $\left[a_{i}: b_{i+h}\right]$ exchanges $x_{a_{i}}=1$ with $x_{b_{i+h}}=0$ if and only if $m_{2}+h \leq n-i<m_{1}$. Therefore ones $\left(y_{A}\right)=m_{1}-\left(m_{1}-m_{2}-h\right)=m_{2}+h$ and ones $\left(y_{B}\right)=m_{2}+\left(m_{1}-m_{2}-h\right)=m_{1}-h$.

According to the definition of $M_{k}$, it consists of three stages $T_{1}^{k}, T_{2}^{k}, T_{3}^{k}$, where $T_{i}^{k}=$ $\cup\left\{S_{k, i+3 j}: 0 \leq j \leq\left\lfloor\frac{2 k-i-3}{3}\right\rfloor\right\}$ (sets $S_{j}$ are defined in Def. 11). Using the notation from Fact 4 , the following fact is an easy consequence of Definition 1

Fact 5 Let $L_{i}=C_{i}$ and $R_{i}=C_{b_{k}-i+1}$ denote the corresponding left and the right columns of registers, and $h_{i}=2^{k-i-1}-1, i=1, \ldots, \frac{b_{k}}{2}$. Then

(i) $\operatorname{regs}\left(S_{k, 1}\right) \subseteq L_{1} \cup R_{1}$ and $S_{k, 1}=S_{R_{1}-\left\{N_{k}\right\}, L_{1}-\{1\}, 0}$

(ii) $\operatorname{regs}\left(S_{k, 2 j}\right) \subseteq L_{j} \cup R_{j}$ and $S_{k, 2 j}=S_{L_{j}, R_{j}, h_{j}}$, for any $j=1, \ldots, \frac{b_{k}}{2}$

(iii) $\operatorname{regs}\left(S_{k, 2 j+1}\right) \subseteq L_{j} \cup L_{j+1} \cup R_{j+1} \cup R_{j}$ and $S_{2 j+1}=S_{L_{j}, L_{j+1}, 0} \cup S_{R_{j+1}, R_{j}, 0}$, for any $j=1, \ldots, \frac{b_{k}}{2}-1$

(iv) $\operatorname{regs}\left(S_{k, 2 k-3}\right) \subseteq L_{k-2} \cup R_{k-2}$ and $S_{k, 2 k-3}=S_{L_{k-2}, R_{k-2}, 0}$

(v) if $\left(L_{j} \cup R_{j}\right) \cap \operatorname{regs}\left(S_{k, i}\right) \neq \emptyset$ then $2 j-1 \leq i \leq 2 j+1$, for any $j=1, \ldots, \frac{b_{k}}{2}-1$

Lemma 1. If the initial content of registers is a 2-sorted 0-1 sequence $x$ then after each stage of multi-pass computation of $M_{k}=T_{1}^{k}, T_{2}^{k}, T_{3}^{k}$ the content of each column $C_{j}$, $j=1, \ldots, b_{k}$, is sorted, that is, each $\left(x^{(p, i)}\right)_{C_{j}}$ is of the form $0^{*} 1^{*}, p=0, \ldots, i=1,2,3$.

Proof. By induction it suffices to prove that for each sequence $y \in \Sigma^{N_{k}}$ with sorted columns $C_{j}, j=1, \ldots, b_{k}$, the outputs $z_{i}=T_{i}^{k}(y), i=1,2,3$ have also the columns sorted. Since each $T_{i}^{k}$, as a mapping, is a composition of mapping $S_{i+3 j}, 0 \leq j \leq\left\lfloor\frac{2 k-i-3}{3}\right\rfloor$, each of which, due to Facts 4 and [5, transforms sorted columns into sorted columns, the lemma follows.

From now on, instead of looking at 0-1 sequences with sorted columns, we will analyze the computations of $M_{k}$ on sequences of integers $\bar{c}=\left(c_{1}, \ldots, c_{b_{k}}\right)$, where $c_{t}$, $t=1, \ldots, b_{k}$, denote the number of ones in a sorted column $C_{t}$. Transformations of $0-1$ sequences defined by sets $S_{j}, j=1, \ldots, 2 k-3$ will be represented by the following mappings:

Definition 3. Let $k \geq 3, h_{i}=2^{k-i-1}-1$ for $i=1, \ldots, k-2$ and $b_{k}=2(k-2)$. The functions dec $_{i}^{k}$, mov ${ }_{i}^{k}$ and cyck over sequences of $b_{k}$ reals are defined as follows. Let $\bar{c}=\left(c_{1}, \ldots, c_{b_{k}}\right)$ and $t \in\left\{1, \ldots, b_{k}\right\}$.

$$
\left(\operatorname{dec}_{i}^{k}(\bar{c})\right)_{t}= \begin{cases}\min \left(c_{i}, c_{b_{k}-i+1}+h_{i}\right) & \text { if } t=i \\ \max \left(c_{i}-h_{i}, c_{b_{k}-i+1}\right) & \text { if } t=b_{k}-i+1 \\ c_{t} & \text { otherwise }\end{cases}
$$




$$
\begin{aligned}
\left(\operatorname{mov}_{i}^{k}(\bar{c})\right)_{t} & = \begin{cases}\min \left(c_{t}, c_{t+1}\right) & \text { if } t=i \text { or } t=b_{k}-i \\
\max \left(c_{t-1}, c_{t}\right) & \text { if } t=i+1 \text { or } t=b_{k}-i+1 \\
c_{t} & \text { otherwise }\end{cases} \\
\left(\operatorname{cyc}^{k}(\bar{c})\right)_{t} & = \begin{cases}\max \left(c_{1}, c_{b_{k}}-1\right) & \text { if } t=1 \\
\min \left(c_{1}+1, c_{b_{k}}\right) & \text { if } t=b_{k} \\
c_{t} & \text { otherwise }\end{cases}
\end{aligned}
$$

Fact 6 Let $x \in \Sigma^{N_{k}}$ be a 0-1 sequence with sorted columns $C_{1}, \ldots, C_{b_{k}}$, let $c_{i}=$ ones $\left(x_{C_{i}}\right)$ and $\bar{c}=\left(c_{1}, \ldots, c_{b_{k}}\right)$. Let $y_{j}=S_{k, j}(x), d_{j, i}=$ ones $\left(\left(y_{j}\right)_{C_{i}}\right)$ and $\overline{d_{j}}=\left(d_{j, 1}, \ldots, d_{j, b_{k}}\right)$, where $i=1, \ldots, b_{k}$ and $j=1, \ldots, 2 k-3$. Then

(i) $\overline{d_{1}}=c y c^{k}(\bar{c})$

(ii) $\overline{d_{2 j}}=\operatorname{dec}_{j}^{k}(\bar{c})$, for any $j=1, \ldots, \frac{b_{k}}{2}$

(iii) $\overline{d_{2 j+1}}=\operatorname{mov}_{j}^{k}(\bar{c})$, for any $j=1, \ldots, \frac{b_{k}}{2}$

Proof. Generally, the fact follows from Fact 5 and the part (ii) of Fact 4 We prove only its parts (i) and (ii). Part (iii) can be proved in the similar way.

(i) Observe that $y_{1}=S_{k, 1}(x)=S_{R_{1}-\left\{N_{k}\right\}, L_{1}-\{1\}, 0}(x)$ due to Fact 5 (ii). It follows that only the content of columns $L_{1}=C_{1}$ and $R_{1}=C_{b_{k}}$ can change, but they remain sorted (according to Lemma 1). Using Fact 4(ii) we have: $m_{1}=$ ones $\left(x_{R_{1}-\left\{N_{k}\right\}}\right)=c_{b_{k}}-x_{N_{k}}$, $m_{2}=\operatorname{ones}\left(x_{L_{1}-\{1\}}\right)=c_{1}-x_{1}$ and

$$
\begin{aligned}
d_{1,1} & =\max \left(m_{1}, m_{2}\right)+x_{1}=\max \left(c_{b_{k}}-x_{N_{k}}+x_{1}, c_{1}\right), \\
d_{1, b_{k}} & =\min \left(m_{1}, m_{2}\right)+x_{N_{k}}=\min \left(c_{b_{k}}, c_{1}+x_{N_{k}}-x_{1}\right) .
\end{aligned}
$$

Now let us consider the following three cases of values $x_{1}$ and $x_{N_{k}}$ :

Case $x_{1}=0$ and $x_{N_{k}}=1$. Then $d_{1,1}=\max \left(c_{b_{k}}-1, c_{1}\right)=c y c^{k}(\bar{c})_{1}$ and $d_{1, b_{k}}=\min \left(c_{b_{k}}, c_{1}+\right.$ $1)=\operatorname{cyc}^{k}(\bar{c})_{1}$.

Case $x_{1}=1$. Then $c_{1}=n_{k}, c_{b_{k}} \leq n_{k}$ and $c_{b_{k}}-x_{N_{k}} \leq n_{k}-1$. In this case: $d_{1,1}=$ $\max \left(n_{k}, c_{b_{k}}-x_{N_{k}}+1,\right)=n_{k}=\max \left(c_{1}, c_{b_{k}}-1\right)$ and $d_{1, b_{k}}=\min \left(n_{k}-1+x_{N_{k}}, c_{b_{k}}\right)=$ $c_{b_{k}}=\min \left(c_{1}+1, c_{b_{k}}\right)$.

Case $x_{N_{k}}=0$. Then $c_{b_{k}}=0$ and $c_{1}-x_{1} \geq 0$. In this case: $d_{1,1}=\max \left(c_{1}, x_{1}\right)=c_{1}=$ $\max \left(c_{1}, c_{b_{k}}-1\right)$ and $d_{1, b_{k}}=\min \left(c_{1}-x_{1}, c_{b_{k}}\right)=c_{b_{k}}=\min \left(c_{1}+1, c_{b_{k}}\right)$.

(ii) We fix any $j \in\left\{1, \ldots, \frac{b_{k}}{2}\right\}$ and observe that $y_{2 j}=S_{2 j}(x)=S_{L_{j}, R_{j}, h_{j}}(x)$ due to Fact 5 (ii). It follows that only the content of columns $L_{j}=c_{j}$ and $R_{j}=c_{b_{k}-j+1}$ can change, but they remain sorted (according to Lemma 1). Using Fact 4 (ii) we have:

$$
\begin{aligned}
d_{2 j, j} & =\text { ones }\left(\left(y_{2 j}\right)_{L_{j}}\right)=\min \left(c_{j}, c_{b_{k}-j+1}+h_{j}\right)=\left(\operatorname{dec}_{j}^{k}(\bar{c})\right)_{j}, \\
d_{2 j, b_{k}-j+1} & =\text { ones }\left(\left(y_{2 j}\right)_{R_{j}}\right)=\max \left(c_{j}-h_{j}, c_{b_{k}-j+1}\right)=\left(\operatorname{dec}_{j}^{k}(\bar{c})\right)_{b_{k}-j+1} .
\end{aligned}
$$

Definition 4. Let $k \geq 3$. Let $Q_{1}^{k}, Q_{2}^{k}$ and $Q_{3}^{k}$ denote the following sets of functions.

$$
\begin{aligned}
& Q_{1}^{k}=\left\{\operatorname{syc}^{k}\right\} \cup\left\{\operatorname{dec}_{3 i-1}^{k}\right\}_{i=1}^{\left\lfloor\frac{k-1}{3}\right\rfloor} \cup\left\{\operatorname{mov}_{3 i}^{k}\right\}_{i=1}^{\left\lfloor\frac{k-2}{3}\right\rfloor} \\
& Q_{2}^{k}=\left\{\operatorname{dec}_{3 i-2}^{k}\right\}_{i=1}^{\left\lfloor\frac{k}{3}\right\rfloor} \cup\left\{\operatorname{mov}_{3 i-1}^{k}\right\}_{i=1}^{\left\lfloor\frac{k-1}{3}\right\rfloor} \\
& Q_{3}^{k}=\left\{\operatorname{dec}_{3 i}^{k}\right\}_{i=1}^{\left\lfloor\frac{k-2}{3}\right\rfloor} \cup\left\{\operatorname{mov}_{3 i-2}^{k}\right\}_{i=1}^{\left\lfloor\frac{k}{3}\right\rfloor}
\end{aligned}
$$


Let us observe that each function in $Q_{i}^{k}, i=1,2,3$, can only modify a few positions in a given sequence of numbers. Moreover, different functions in $Q_{i}^{k}$ can only modify disjoint sets of positions. For a function $f: R^{m} \mapsto R^{m}$ let us define

$$
\operatorname{args}(f)=\left\{i \in\{1, \ldots, m\}: \exists_{\bar{c} \in R^{m}}(f(\bar{c}))_{i} \neq(\bar{c})_{i}\right\}
$$

The following facts formalize our observations.

Fact $7 \operatorname{args}\left(c y c^{k}\right)=\left\{1, b_{k}\right\}, \operatorname{args}\left(\operatorname{dec}_{i}^{k}\right)=\left\{i, b_{k}-i+1\right\}, \operatorname{args}\left(\operatorname{mov}_{i}^{k}\right)=\left\{i, i+1, b_{k}-\right.$ $\left.i, b_{k}-i+1\right\}$, where $i=1, \ldots, k-2$.

Fact 8 For each pair of functions $f, g \in Q_{i}^{k}, f \neq g, i=1,2,3$, we have

(i) $\operatorname{args}(f) \cap \operatorname{args}(g)=\emptyset$;

(ii) for any $\bar{c}=\left(c_{1}, \ldots, c_{b_{k}}\right)$ and $j \in\left\{1, \ldots, b_{k}\right\}$

$$
(f(g(\bar{c})))_{j}= \begin{cases}(f(\bar{c}))_{j} & \text { if } j \in \operatorname{args}(f) \\ (g(\bar{c}))_{j} & \text { if } j \in \operatorname{args}(g) \\ c_{j} & \text { otherwise }\end{cases}
$$

Corollary 1. Each set $Q_{i}^{k}, i=1,2,3$, uniquely determines a mapping, in which functions from $Q_{i}^{k}$ can be apply in any order. Moreover, if $f \in Q_{i}^{k}, \bar{c} \in R^{b_{k}}$ and $j \in \operatorname{args}(f)$ then $\left(Q_{i}^{k}(\bar{c})\right)_{j}=(f(\bar{c}))_{j}$.

We would like to prove that the result of applying $Q_{i}^{k}, i=1,2,3$, to a sequence $\bar{c}=$ $\left(c_{1}, \ldots, c_{b_{k}}\right)$ of numbers of ones in columns $C_{1}, \ldots, C_{b_{k}}$ is equivalent to applying the set of comparators $T_{i}^{k}$ to the content of registers, if each column is sorted.

Lemma 2. Let $x \in \Sigma^{N_{k}}$ be a 0-1 sequence with sorted columns $C_{1}, \ldots, C_{b_{k}}$, let $c_{i}=$ ones $\left(x_{C_{i}}\right)$ and $\bar{c}=\left(c_{1}, \ldots, c_{b_{k}}\right)$. Let $y_{j}=T_{j}^{k}(x), d_{j, i}=$ ones $\left(\left(y_{j}\right)_{C_{i}}\right)$ and $\overline{d_{j}}=\left(d_{j, 1}, \ldots, d_{j, b_{k}}\right)$, where $i=1, \ldots, b_{k}$ and $j=1,2,3$. Then $Q_{j}^{k}(\bar{c})=\overline{d_{j}}$.

Proof. Recall that $T_{j}^{k}=\bigcup\left\{S_{k, j+3 i}: 0 \leq i \leq \frac{2 k-j-3}{3}\right\}$. For a set of comparators $S$ let us define

$$
\operatorname{cols}(S)=\left\{i \in\left\{1, \ldots, b_{k}\right\}: \operatorname{regs}(S) \cap C_{i} \neq \emptyset\right\} .
$$

From Fact 5 i-iv) it follows that $\operatorname{cols}\left(S_{k, 1}\right)=\left\{1, b_{k}\right\}$ and for $i=1, \ldots, k-2 \operatorname{cols}\left(S_{k, 2 i}\right)=$ $\left\{i, b_{k}-i+1\right\}$ and $\operatorname{cols}\left(S_{k, 2 i+1}\right)=\left\{i, i+1, b_{k}-i, b_{k}-i+1\right\}$. From Fact $5(\mathrm{v})$ we get that $\operatorname{cols}\left(S_{k, j+3 i}\right) \cap \operatorname{cols}\left(S_{k, j+3 i^{\prime}}\right)=\emptyset$ if $i \neq i^{\prime}$. Thus we can observe a 1-1 correspondence between a function $f$ in $Q_{j}^{k}$ and a set of comparators $S_{k, j+3 i} \subseteq T_{j}^{k}$ such that $\operatorname{args}(f)=$ $\operatorname{cols}\left(S_{k, j+3 i}\right)$ Then for each $t \in \operatorname{args}(f)\left(Q_{j}^{k}(\bar{c})\right)_{t}=(f(\bar{c}))_{t}=\left(\overline{d_{j}}\right)_{t}$, as the consequence of Corollary 1 and Fact 6 .

Definition 5. We say that a sequence of numbers $\bar{c}=\left(c_{1}, \ldots, c_{2 m}\right)$ is flat if $c_{1} \leq c_{2} \leq$ $\ldots, c_{2 m} \leq c_{1}+1$. We say that a sequence $\bar{c}$ is 2 -flat if subsequences $\left(c_{1}, c_{3}, \ldots, c_{2 m-1}\right)$ and $\left(c_{2}, c_{4}, \ldots, c_{2 m}\right)$ are flat. We say that $\bar{c}$ is balanced if $c_{i}+c_{2 m-i+1}=c_{1}+c_{2 m}$, for $i=2, \ldots, m$. For a balanced sequence $\bar{c}$ define height $(\bar{c})$ as $c_{1}+c_{2 m}$. 
Proposition 3. Let $k \geq 3, x \in \Sigma^{N_{k}}, \bar{c}=\left(c_{1}, \ldots, c_{b_{k}}\right)$, where $c_{i}=$ ones $\left(x_{C_{i}}\right)\left(C_{i}\right.$ is as usual a column in the matrix of registers), $i=1, \ldots, b_{k}$. Then

1. $x$ is sorted if and only if columns of $x$ are sorted and $\bar{c}$ is flat;

2. $x$ is 2-sorted if and only if columns of $x$ are sorted and $\bar{c}$ is 2-flat;

Now we are ready to reduce the proof of Theorem 2 to the proof of following lemma.

Lemma 3. Let $k \geq 3$. If for each 2-flat sequence $\bar{c}=\left(c_{1}, \ldots, c_{b_{k}}\right)$ of integers from $\left[0,2^{k-1}-1\right]$ the result of application $\left(Q_{3}^{k} \circ Q_{2}^{k} \circ Q_{1}^{k}\right)^{2 k-5}$ to $(\bar{c})$ is a flat sequence, then $M_{k}$ is a $2 k-5$-pass merger of two sorted sequences given in odd and even registers, respectively.

Proof. Assume that for each 2-flat sequence $\bar{c}=\left(c_{1}, \ldots, c_{b_{k}}\right)$ the result of application $\left(Q_{3}^{k} \circ Q_{2}^{k} \circ Q_{1}^{k}\right)^{2 k-5}$ to $(\bar{c})$ is a flat sequence. Let $\bar{x} \in \Sigma^{N_{k}}$ be a 2 -sorted sequence and $\bar{c}=\left(c_{1}, \ldots, c_{b_{k}}\right)$, where $c_{i}=$ ones $\left(\bar{x}_{C_{i}}\right)\left(C_{i}\right.$ is as usual a column in the matrix of registers), $i=1, \ldots, b_{k}$. Then $\bar{c}$ is 2 -flat due to Proposition 3 and each $c_{i} \in\left[0,2^{k-1}-1\right]$, because the height of columns is $2^{k-1}-1$. Recall that $\bar{x}^{(j)}=\left(M_{k}\right)^{j}(\bar{x})$ and let $c_{j, i}=\operatorname{ones}\left(\bar{x}_{C_{i}}^{(j)}\right)$. Using Lemma 2 and easy induction we get that the equality $\left(Q_{3}^{k} \circ Q_{2}^{k} \circ Q_{1}^{k}\right)^{j}(\bar{c})=$ $\left(c_{j, 1}, \ldots, c_{j, b_{k}}\right)$ is true for $j=1, \ldots, 2 k-5$. Since $\left(Q_{3}^{k} \circ Q_{2}^{k} \circ Q_{1}^{k}\right)^{2 k-5}(\bar{c})$ is a flat sequence, the sequence $\bar{x}^{(2 k-5)}$ is sorted.

\subsection{Analysis of Balanced Columns}

Due to Lemma 3 we can only analyze the results of periodic application of the functions $Q_{1}^{k}, Q_{2}^{k}$ and $Q_{3}^{k}$ to a sequence of integers representing the numbers of ones in each register column. We know also that an initial sequence is 2 -flat. To simplify our analysis further, we start it with initial values restricted to be balanced 2 -flat sequences. Then we observe that the functions are monotone and any 2-flat sequence can be bounded from below and above by balanced 2-flat sequences whose heights differ only by one.

Lemma 4. Let $k \geq 3$ and $\bar{c}=\left(c_{1}, \ldots, c_{b_{k}}\right)$ be a balanced sequence of numbers. Let $s=$ height $(\bar{c})$ and let $f$ be a function from $Q_{1}^{k} \cup Q_{2}^{k} \cup Q_{3}^{k}$. Then $f(\bar{c})$ is also balanced and height $(f(\bar{c}))=s$.

Proof. Let $\bar{c}$ and $s$ be as in Lemma and let $f(\bar{c})=\left(d_{1}, \ldots, d_{b_{k}}\right)$. The function $f \in Q_{1}^{k} \cup$ $Q_{2}^{k} \cup Q_{3}^{k}$ can be either $c y c^{k}$ or one of $m o v_{j}^{k}$, dec $c_{j}^{k}, j=1, \ldots, k-2$, according to Definition 4 Each of the functions can only modify one or two pairs of positions of the form $\left(i, b_{k}-i+1\right)$ in $\bar{c}$ (see Definition 3). The other pairs are left untouched, so the sum of their values cannot change. In case of $c y c^{k}$ the modified pair is $\left(1, b_{k}\right)$ and $d_{1}+d_{b_{k}}=$ $\max \left(c_{1}, c_{b_{k}}-1\right)+\min \left(c_{1}+1, c_{b_{k}}\right)=s$. In case of $d e c_{j}^{k}$ the pair is $\left(j, b_{k}-j+1\right)$ and $d_{j}+d_{b_{k}-j+1}=\min \left(c_{j}, c_{b_{k}-j+1}+h_{j}\right)+\max \left(c_{j}-h_{j}, c_{b_{k}-j+1}\right)=\min \left(c_{j}-h_{j}, c_{b_{k}-j+1}\right)+$ $h_{j}+\max \left(c_{j}-h_{j}, c_{b_{k}-j+1}\right)=s$. Finally, if $f=m o v_{j}^{k}$ then we have two pairs $\left(j, b_{k}-\right.$ $j+1)$ and $\left(j+1, b_{k}-j\right)$. Then $d_{j}+d_{b_{k}-j+1}=\min \left(c_{j}, c_{j+1}\right)+\max \left(c_{b_{k}-j}, c_{b_{k}-j+1}\right)=$ $\min \left(c_{j}, c_{j+1}\right)+\max \left(s-c_{j+1}, s-c_{j}\right)=s$ and in case of the second pair $d_{j+1}+d_{b_{k}-j}=$ $\max \left(c_{j}, c_{j+1}\right)+\min \left(c_{b_{k}-j}, c_{b_{k}-j+1}\right)=\max \left(c_{j}, c_{j+1}\right)+\min \left(s-c_{j+1}, s-c_{j}\right)=s$. 
It follows from Lemma 4 that if we start the periodical application of the functions $Q_{1}^{k}, Q_{2}^{k}$ and $Q_{3}^{k}$ to a balanced 2-flat initial sequence then it remains balanced after each function application and its height will not changed. Therefore, we can only trace the values in the first half of generated sequences. If needed, a value in the second half can be computed from the height and the corresponding value in the first half. To get a better view on the structure of generated sequences, we subtract half of the height from each element of the initial sequence and proceed with such modified sequences to the end. At the end the subtracted value is added to each element of the final sequence. The following fact justifies the described above procedure.

Fact 9 Let $f$ be a function from $Q_{1}^{k} \cup Q_{2}^{k} \cup Q_{3}^{k}$. Then $f$ is monotone and for each $t \in R$ and $\left(c_{1}, \ldots, c_{b_{k}}\right)$ the following equation is true

$$
f\left(c_{1}-t, \ldots, c_{b_{k}}-t\right)=f\left(c_{1}, \ldots, c_{b_{k}}\right)-(t, \ldots, t) .
$$

Proof. The fact follows from the similar properties of min and max functions: they are monotone and the equations: $\min (x-t, y-t)=\min (x, y)-t$ and $\max (x-t, y-t)=$ $\max (x, y)-t$ are obviously true. Each $f$ in $Q_{1}^{k} \cup Q_{2}^{k} \cup Q_{3}^{k}$ is defined with the help of these simple functions, thus $f$ inherits the properties.

Corollary 2. Let $f=f_{l} \circ f_{l-1} \circ \ldots \circ f_{1}$, where $f_{i} \in\left\{Q_{1}^{k}, Q_{2}^{k}, Q_{3}^{k}\right\}, 1 \leq i \leq l$. Then $f$ is monotone and for any $t \in R$ and $\left(c_{1}, \ldots, c_{b_{k}}\right) \in R^{b_{k}}$

$$
f\left(c_{1}-t, \ldots, c_{b_{k}}-t\right)=f\left(c_{1}, \ldots, c_{b_{k}}\right)-(t, \ldots, t) .
$$

Definition 6. Let $\bar{c}=\left(c_{1}, \ldots, c_{b_{k}}\right) \in R^{b_{k}}$ be a balanced sequence and $s=$ height $(\bar{c})$. We call $\left(c_{1}-\frac{s}{2}, c_{2}-\frac{s}{2}, \ldots, c_{k-2}-\frac{s}{2}\right) \in R^{b_{k} / 2}$ the reduced sequence of $\bar{c}$ and denote it by reduce $(\bar{c})$. For a sequence $\bar{d}=\left(d_{1}, \ldots, d_{k-2}\right) \in R^{k-2}$ we define s-extended sequence $\operatorname{ext}(\bar{d}, s)$ as

$$
\left(d_{1}+\frac{s}{2}, d_{2}+\frac{s}{2}, \ldots, d_{k-2}+\frac{s}{2}, \frac{s}{2}-d_{k-2}, \frac{s}{2}-d_{k-3}, \ldots, \frac{s}{2}-d_{1}\right) .
$$

For any $t \in R$ and a function $f: R^{b_{k}} \mapsto R^{b_{k}}$ that maps each balanced sequence to a balanced one and preserves its height let reduce $(f, t)$ denote a function on $R^{k-2}$ such that $(\operatorname{reduce}(f, t))(\bar{d})=\operatorname{reduce}(f(\operatorname{ext}(\bar{d}, t)))$ for any $\bar{d} \in R^{k-2}$.

Observe that for a balanced sequence $\bar{c}$ with height $s$ the sequence $\operatorname{ext}(\operatorname{reduce}(\bar{c}), s)$ is equal to $\bar{c}$. Moreover, for any $t \in R$ and a sequence $\bar{d} \in R_{k-2}$ the sequence $\operatorname{ext}(\bar{d}, t)$ is balanced and its height is $t$, thus reduce $(\operatorname{ext}(\bar{d}, t))=\bar{d}$. Note also that functions $Q_{1}^{k}, Q_{2}^{k}$ and $Q_{3}^{k}$ preserve the property of being balanced and the sequence height (see Lemma 4), so we can analyze a periodical application of their reduced forms to a reduced balanced 2-flat input.

Fact 10 Let $f=f_{l} \circ f_{l-1} \circ \ldots \circ f_{1}$, where $f_{i} \in\left\{Q_{1}^{k}, Q_{2}^{k}, Q_{3}^{k}\right\}, 1 \leq i \leq l$. Let $\bar{c} \in R^{b_{k}}$ be balanced and $s=$ height $(\bar{c})$ Let $\hat{f}_{i}=\operatorname{reduce}\left(f_{i}, s\right), 1 \leq i \leq l$. Then $f(\bar{c})=\operatorname{ext}\left(\left(\hat{f}_{l} \circ \hat{f}_{l-1} \circ\right.\right.$ $\left.\left.\ldots \circ \hat{f}_{1}\right)(\operatorname{reduce}(\bar{c})), s\right)$. 
Definition 7. Let MinMax $(x, y)=(\min (x, y), \max (x, y)), \operatorname{Min}(x)=\min (x,-x), \operatorname{Cyc}(x)=$ $\max (x,-x-1)$ and $\operatorname{Dec}_{i}(x)=\min \left(x,-x+H_{i}\right)$, where $H_{i}=2^{i}-1, i=1, \ldots$ Moreover, let us define the following sequences of functions:

$$
\begin{aligned}
& \hat{Q}_{1}^{k}=(\text { Cyc }) \oplus \bigoplus_{i=1}^{\left\lfloor\frac{k-3}{3}\right\rfloor}\left(\operatorname{Dec}_{k-3 i}, \text { MinMax }\right) \oplus\left(F_{1}^{k}\right) \\
& \hat{Q}_{2}^{k}=\bigoplus_{i=1}^{\left\lfloor\frac{k-2}{3}\right\rfloor}\left(\operatorname{Dec}_{k-3 i+1}, \text { MinMax }\right) \oplus\left(F_{2}^{k}\right) \\
& \hat{Q}_{3}^{k}=\bigoplus_{i=1}^{\left\lfloor\frac{k-2}{3}\right\rfloor}\left(\text { MinMax }_{1} \operatorname{Dec}_{k-3 i-1}\right) \oplus\left(F_{3}^{k}\right),
\end{aligned}
$$

where $\oplus$ denote concatenation of sequences and for $i=1,2$

$$
F_{i}^{k}=\left\{\begin{array}{ll}
() & \text { if } k \equiv 2 i+1(\bmod 3) \\
\left(\operatorname{Dec}_{1}\right) & \text { if } k \equiv 2 i+2(\bmod 3) \\
\left(\operatorname{Dec}_{2},\right. \text { Min) } & \text { if } k \equiv 2 i(\bmod 3)
\end{array} \quad F_{3}^{k}= \begin{cases}() & \text { if } k \equiv 2(\bmod 3) \\
(\operatorname{Min}) & \text { if } k \equiv 0(\bmod 3) \\
(\text { MinMax }) & \text { if } k \equiv 1(\bmod 3)\end{cases}\right.
$$

Lemma 5. Let $k \geq 3$ and $t \in R$. Then reduce $\left(Q_{i}^{k}, t\right)=\otimes \hat{Q}_{i}^{k}$, where $i=1,2,3$ and $\otimes$ denotes the Cartesian product of a sequence of functions.

Proof. Let $k \geq 3, i \in\{1,2,3\}$ and $t \in R$. Let $\bar{d} \in R^{k-2}$. By Def.6 $\left(\right.$ reduce $\left.\left(Q_{i}^{k}, t\right)\right)(\bar{d})=$ reduce $\left(Q_{i}^{k}(\operatorname{ext}(\bar{d}, t))\right)$. Let $\bar{e}=\operatorname{ext}(\bar{d}, t)=\left(d_{1}+\frac{t}{2}, \ldots, d_{k-2}+\frac{t}{2},-d_{k-2}+\frac{t}{2}, \ldots,-d_{1}+\right.$ $\left.\frac{t}{2}\right)$. The sequence $\bar{e}$ is balanced and height $(\bar{e})=t$. To get the lemma we would like to prove that for $j=1, \ldots, k-2$ the equalities $\left(Q_{i}^{k}(\bar{e})\right)_{j}-\frac{t}{2}=\left(\left(\otimes \hat{Q}_{i}^{k}\right)(\bar{d})\right)_{j}$ hold. The proof is by case analysis of values of $i$ and $j$. In the following equations we use Definitions 3 , 4,6and7

1. (Case: $i=1$ and $j=1)$. Then $\left(Q_{1}^{k}(\bar{e})\right)_{1}=\left(c y c^{k}(\bar{e})\right)_{1}=\max \left(d_{1}+\frac{t}{2},-d_{1}+\frac{t}{2}-1\right)=$ $\max \left(d_{1},-d_{1}-1\right)+\frac{t}{2}=C y c\left(d_{1}\right)+\frac{t}{2}=\left(\left(\otimes \hat{Q}_{i}^{k}\right)(\bar{d})\right)_{1}+\frac{t}{2}$.

2. (Case: $i+j>2$ and $i+j \equiv 0(\bmod 3))$. Let $l$ be such that $j=3 l-i$. Then $\left(Q_{i}^{k}(\bar{e})\right)_{j}=$ $\left(\operatorname{dec}_{3 l-i}^{k}(\bar{e})\right)_{3 l-i}=\min \left(d_{3 l-i}+\frac{t}{2},-d_{3 l-i}+\frac{t}{2}+2^{k-(3 l-i)-1}-1\right)=\min \left(d_{3 l-i},-d_{3 l-i}+\right.$ $\left.H_{k-(3 l-i)-1}\right)+\frac{t}{2}=\operatorname{Dec}_{k-3 l+i-1}\left(d_{3 l-i}\right)+\frac{t}{2}=\left(\left(\otimes \hat{Q}_{i}^{k}\right)(\bar{d})\right)_{j}+\frac{t}{2}$.

3. (Case: $i+j>2, j<k-2$ and $i+j \equiv 1(\bmod 3))$. Let $l$ be such that $j=3 l-$ $i+1$. Then $\left(Q_{i}^{k}(\bar{e})\right)_{j}=\left(\operatorname{mov}_{3 l-i+1}^{k}(\bar{e})\right)_{3 l-i+1}=\min \left(d_{3 l-i+1}+\frac{t}{2}, d_{3 l-i+2}+\frac{t}{2}\right)=$ $\min \left(d_{3 l-i+1}, d_{3 l-i+2}\right)+\frac{t}{2}$. Starting from the other side we get $\left(\left(\otimes \hat{Q}_{i}^{k}\right)(\bar{d})\right)_{3 l-i+1}=$ $\left(\operatorname{MinMax}\left(d_{3 l-i+1}, d_{3 l-i+2}\right)\right)_{1}=\min \left(d_{3 l-i+1}, d_{3 l-i+2}\right)$ and we are done.

4. (Case: $i+j>2, j=k-2$ and $i+j \equiv 1(\bmod 3))$. Let $l$ be as in previous case. Then $\left(Q_{i}^{k}(\bar{e})\right)_{k-2}=\left(\operatorname{mov}_{k-2}^{k}(\bar{e})\right)_{k-2}=\min \left(d_{k-2}+\frac{t}{2},-d_{k-2}+\frac{t}{2}\right)=\min \left(d_{k-2},-d_{k-2}\right)+$ $\frac{t}{2}=\operatorname{Min}\left(d_{k-2}\right)+\frac{t}{2}=\left(\left(\otimes \hat{Q}_{i}^{k}\right)(\bar{d})\right)_{k-2}+\frac{t}{2}$.

5. (Case: $i+j>2$ and $i+j \equiv 2(\bmod 3))$. Let $l$ be such that $j=3 l-i+2$. Then $\left(Q_{i}^{k}(\bar{e})\right)_{3 l-i+2}=\left(\operatorname{mov}_{3 l-i+1}^{k}(\bar{e})\right)_{3 l-i+2}=\max \left(d_{3 l-i+1}+\frac{t}{2}, d_{3 l-i+2}+\frac{t}{2}\right)=$ $\max \left(d_{3 l-i+1}, d_{3 l-i+2}\right)+\frac{t}{2}$. Starting from the other side we get $\left(\left(\otimes \hat{Q}_{i}^{k}\right)(\bar{d})\right)_{3 l-i+2}=$ $\left(\operatorname{MinMax}\left(d_{3 l-i+1}, d_{3 l-i+2}\right)\right)_{2}=\max \left(d_{3 l-i+1}, d_{3 l-i+2}\right)$ and we are finally done. 
Instead of tracing individual values in reduced sequences after each application of a function from $\left\{\otimes \hat{Q}_{1}^{k}, \otimes \hat{Q}_{2}^{k}, \otimes \hat{Q}_{3}^{k}\right\}$ we will trace intervals in which the values should be and observe how the lengths of intervals are decreasing during the computation. So let us now define the intervals and show a fact about computations on them.

Definition 8. Let $k \geq 3, H_{i}=2^{i}-1$ for $1 \leq i \leq k-1$. Let $I(0)$ denote the interval $\left[-\frac{1}{2}, 0\right]$ and, in similar way, let $I(i)=\left[-\frac{1}{2}, \frac{H_{i}}{2}\right], 1 \leq i \leq k-1, I(-k)=\left[-\frac{H_{k-1}}{2}, 0\right]$ and $I( \pm k)=\left[-\frac{H_{k-1}}{2}, \frac{H_{k-1}}{2}\right]$. Moreover, we will write $I\left(w_{1}, w_{2}, \ldots, w_{l}\right)$ for the Cartesian product $I\left(w_{1}\right) \times I\left(w_{2}\right) \times \ldots \times I\left(w_{l}\right)$, where each $w_{i} \in\{0,1,2, \ldots, k-1,-k, \pm k\}$.

Fact 11 The following inclusions are true:

1. $\operatorname{Dec}_{i}(I(i+1)) \subseteq I(i)$ and $D e c_{i}(I(w)) \subseteq I(w)$, for $1 \leq i \leq k-2$ and $w \in\{0,-k, \pm k\}$;

2. $C y c(I(-k)) \subseteq I(k-1)$ and $C y c(w) \subseteq C y c(w)$, for $w \in\{0, k-1\}$;

3. $\operatorname{Min}(I( \pm k)) \subseteq I(-k)$ and $\operatorname{Min}(I(1)) \subseteq I(0)$;

4. $\operatorname{MinMax}(I( \pm k,-k)) \subseteq(I(-k, \pm k))$;

5. $\operatorname{MinMax}(I(i, w)) \subseteq(I(w, i))$, for $1 \leq i \leq k-1$ and $w \in\{0,-k\}$.

Proof. The proof of each inclusion is a straightforward consequence of the definitions of a given function and intervals. Therefore we check only inclusions given in the first item. Let $x \in I(i+1)=\left[-\frac{1}{2}, \frac{H_{i+1}}{2}\right]$. If $x \in I(i)=\left[-\frac{1}{2}, \frac{H_{i}}{2}\right]$. then $\operatorname{Dec}_{i}(x)=\min (x,-x+$ $\left.H_{i}\right)=x$ since $2 x \leq H_{i}$. Otherwise $x$ must be in $\left(\frac{H_{i}}{2}, \frac{H_{i+1}}{2}\right]$, but then $x>-x+H_{i}$ and $\operatorname{Dec}_{i}(x)=-x+H_{i} \in\left[-\frac{1}{2}, \frac{H_{i}}{2}\right)$ since $H_{i+1}=2 H_{i}+1$.

To proof the second inclusion for $\operatorname{Dec}_{i}$ let us observe that if $x \leq 0$ then $\operatorname{Dec}_{i}(x)=x$. It follows that $\operatorname{Dec}_{i}(I(0)) \subseteq I(0)$ and $\operatorname{Dec}_{i}(I(-k)) \subseteq I(-k)$. In case of $x \in I( \pm k)$ we only have to check the positive values of $x$. such that $x \geq-x+H_{i}$. But then $\operatorname{Dec}_{i}(x)=$ $-x+H_{i}>-x$ and both $x,-x \in I( \pm k)$.

Now we are ready to define sequences of intervals that are used to describe states of computation after each periodic application of functions $\hat{Q}_{1}^{k}, \hat{Q}_{2}^{k}$ and $\hat{Q}_{3}^{k}$ to a reduced sequence of numbers of ones in columns.

Definition 9. Let $k \geq 3$. By $Z^{k}$ we denote the sequence $(0,0,0)^{\left\lceil\frac{k-2}{3}\right\rceil}$ and, in the similar way, $U_{1}^{k}=( \pm k, \pm k,-k)^{\left\lceil\frac{k-2}{3}\right\rceil}, U_{2}^{k}=( \pm k,-k, \pm k)^{\left\lceil\frac{k-2}{3}\right\rceil}$ and $U_{0}^{k}=(-k, \pm k, \pm k)^{\left\lceil\frac{k-2}{3}\right\rceil}$.

Next, let $V_{1}^{k}=\bigoplus_{i=1}^{\left\lceil\frac{k-2}{3}\right\rceil}(k-3 i+2, k-3 i,-k), V_{2}^{k}=\bigoplus_{i=1}^{\left\lceil\frac{k-2}{3}\right\rceil}(k-3 i+1,-k, k-3 i)$ and let $V_{0}^{k}=\bigoplus_{i=1}^{\left\lceil\frac{k-2}{3}\right\rceil}(-k, k-3 i+1, k-3 i-1)$.

Finally, let $W_{1}^{k}=\bigoplus_{i=1}^{\left\lceil\frac{k-2}{3}\right\rceil}(k-3 i+2, k-3 i, 0), W_{2}^{k}=\bigoplus_{i=1}^{\left\lceil\frac{k-2}{3}\right\rceil}(k-3 i+1,0, k-3 i)$ and let $W_{0}^{k}=\bigoplus_{i=1}^{\left\lceil\frac{k-2}{3}\right\rceil}(0, k-3 i+1, k-3 i-1)$.

Note that all sequences defined above are of length $3\left\lceil\frac{k-2}{3}\right\rceil \geq k-2$ and their elements are interval descriptors as defined in Definition 8

Definition 10. Let $k \geq 3$. Let $\bar{a}=\left(a_{1}, \ldots, a_{n}\right)$ and $\bar{b}=\left(b_{1}, \ldots, b_{n}\right)$ be any sequences, where $n \geq k-2$. For $0 \leq i \leq k-2$ let join $k(i, \bar{a}, \bar{b})$ denote $\left(a_{1}, \ldots, a_{i}, b_{i+1}, \ldots, b_{k-2}\right)$. 
Definition 11. Let $k \geq 3$. Let $X_{i}^{k}$ denote a state sequence after $i$ stages and be defined as:

$$
X_{i}^{k}= \begin{cases}\operatorname{join}_{k}\left(\left\lceil\frac{i+1}{2}\right\rceil, V_{i \text { mod } 3}^{k}, U_{i \text { mod } 3}^{k}\right) & \text { for } i=1, \ldots, 2 k-5 \\ \operatorname{join}_{k}\left(3 k-6-i, V_{i \text { mod } 3}, W_{i \text { mod } 3}^{k}\right) & \text { for } i=2 k-4, \ldots, 3 k-7 \\ \operatorname{join}_{k}\left(\left\lceil\frac{i+1-(3 k-6)}{2}\right\rceil, Z^{k}, W_{i \text { mod } 3}^{k}\right) & \text { for } i=3 k-6, \ldots, 5 k-12\end{cases}
$$

For example, to create $X_{1}^{k}$ we take the first element of $V_{1}^{k}$ and the rest of elements from $U_{1}^{k}$ obtaining the sequence $(k-1, \pm k,-k, \pm k, \pm k,-k, \pm k, \pm k,-k, \ldots)$ of length $k-2$. In the next lemma we claim that $X_{1}^{k}$ really describes the state after the first stage of computation, where input is a balanced 2-flat sequence.

Lemma 6. Let $k \geq 3$ and let $\bar{c}=\left(c_{1}, \ldots, c_{b_{k}}\right)$ be a balanced 2 -flat sequence of integers from $\left[0,2^{k-1}-1\right]$. Let $s=$ height $(\bar{c})$ and let $\bar{d}=$ reduce $(\bar{c})$. Then $\left(\otimes \hat{Q}_{1}^{k}\right)(\bar{d}) \in I\left(X_{1}^{k}\right)$.

Proof. Recall that $H_{i}=2^{i}-1$. Let $\bar{d}=\left(d_{1}, \ldots, d_{k-2}\right)$ By Definitions 5 and $6 s=c_{i}+$ $c_{b_{k}-i+1}$ and each $d_{i}=c_{i}-\frac{s}{2}=\frac{c_{i}-c_{b_{k}-i+1}}{2}$. Observe that each $d_{i} \in I( \pm k)=\left[-\frac{H_{k-1}}{2}, \frac{H_{k-1}}{2}\right]$. It follows from the following sequence of inequalities: $-\frac{H_{k-1}}{2} \leq \frac{-c_{b_{k}-i+1}}{2} \leq \frac{c_{i}-c_{b_{k}}-i+1}{2} \leq$ $\frac{c_{i}}{2} \leq \frac{H_{k-1}}{2}$. Moreover, the sequence $\bar{d}$ is 2-flat, because $\bar{c}$ is 2 -flat. That means that $d_{1} \leq$ $d_{3} \leq d_{5} \leq \ldots \leq d_{k^{\prime}} \leq d_{1}+1$ and $d_{2} \leq d_{4} \leq d_{6} \leq \ldots \leq d_{k^{\prime \prime}} \leq d_{2}+1$, where $k^{\prime}=2\left\lceil\frac{k-2}{2}\right\rceil-$ 1 and $k^{\prime \prime}=2\left\lfloor\frac{k-2}{2}\right\rfloor$.

Fact 12 Either $-\frac{1}{2} \leq d_{1}$ and $d_{k^{\prime \prime}} \leq 0$ or $-\frac{1}{2} \leq d_{2}$ and $d_{k^{\prime}} \leq 0$.

To prove the fact we consider three cases of the value of $d_{1}$. Case $d_{1} \geq 0$ : In this case we only have to prove that $d_{k^{\prime \prime}} \leq 0$. But it is true since $d_{k^{\prime \prime}}=$ $\frac{c_{k^{\prime \prime}}-c_{b_{k}}-k^{\prime \prime}+1}{2} \leq \frac{c_{b_{k}}-c_{1}}{2}=-d_{1} \leq 0$. The last inequality holds, because $\bar{c}$ is 2 -flat and both $k^{\prime \prime}$ and $b_{k}$ are even.

Case $d_{1} \leq-1$. Then $d_{k^{\prime}} \leq d_{1}+1 \leq 0$. Thus we have only to prove that $d_{2} \geq-\frac{1}{2}$. Similar to the previous case, we observe that $d_{2}=\frac{c_{2}-c_{b_{k}-1}}{2} \geq \frac{c_{b_{k}}-1-\left(c_{1}+1\right)}{2}=-d_{1}-1 \geq 0$.

Case $d_{1}=-\frac{1}{2}$ : Then $d_{k^{\prime}} \leq d_{1}+1=\frac{1}{2}$ and from $-\frac{1}{2}=\frac{c_{1}-c_{b_{k}}}{2}$ we get $c_{1}+1=c_{b_{k}} \leq$ $c_{2}+1$. Since $c_{2} \geq c_{1}$, we have $d_{2} \geq d_{1}=-\frac{1}{2}$. If $d_{k^{\prime}} \leq 0$, we are done. Otherwise $d_{k^{\prime}}=\frac{1}{2}$ and we have to show that $d_{k^{\prime \prime}} \leq 0$. To this end let us notice that $\frac{s}{2}=c_{1}-d_{1}=c_{1}+\frac{1}{2}$ and $c_{b_{k}-k^{\prime}+1}=s-c_{k^{\prime}}=s-\left(d_{k^{\prime}}+\frac{s}{2}\right)=\frac{s}{2}-\frac{1}{2}=c_{1}$. It follows that $c_{k^{\prime \prime}}=c_{1}$ since $c_{1} \leq$ $c_{2} \leq c_{k^{\prime \prime}} \leq c_{b_{k}-k^{\prime}+1}=c_{1}$. Thus $d_{k^{\prime \prime}}=d_{1}=-\frac{1}{2}$ and this concludes the proof of Fact 12 ,

From Fact 12 and since $\bar{d}$ is 2-flat we can immediately get the following corollary.

Corollary 3. $\bar{d} \in I((k-1,-k, k-1,-k, \ldots) \cup I(-k, k-1,-k, k-1, \ldots)$.

To finish the proof of the lemma we need one more fact:

Fact $13\left(\otimes \hat{Q}_{1}^{k}\right)\left(I((k-1,-k, k-1,-k, \ldots) \cup I(-k, k-1,-k, k-1, \ldots)) \subseteq I\left(X_{1}^{k}\right)\right.$.

To prove this fact let us firstly represent $X_{1}^{k}$ in the same form as $\hat{Q}_{1}^{k}$ is.

$$
X_{1}^{k}=(k-1) \oplus \bigoplus_{i=1}^{\left\lfloor\frac{k-3}{3}\right\rfloor}( \pm k,-k, \pm k) \oplus\left(Y_{1}^{k}\right)
$$


where $Y_{1}^{k}$ is empty if $k \equiv 0(\bmod 3), Y_{1}^{k}=( \pm k)$ if $k \equiv 1(\bmod 3)$ and $Y_{1}^{k}=( \pm k,-k)$ if $k \equiv 2(\bmod 3)$. Looking now at both representations we can see that the output of $C y c$ function should be in $I(k-1)$, the output of each $D e c_{i}$ function should be in $I( \pm k)$ and the output of MinMax should be in $I(-k) \times I( \pm k)$. If Min function is used, then its output should be in $I(-k)$. The input to $C y c$ is either from $I(k-1)$ or from $I(-k)$. In both cases we get desired output according to Fact 112. In the similar way, the input to each $D e c_{i}$ function is either from $I(k-1) \subseteq I( \pm k)$ or from $I(-k) \subseteq I( \pm k)$. But $\operatorname{Dec}_{i}(I( \pm k)) \subseteq I( \pm k)$ by Fact 11, 1 . From Fact 113 we have $\operatorname{Min}(I( \pm k)) \subseteq I(-k)$. Finally, the input to MinMax function is either from $I(k-1) \times I(-k)$ or from $I(-k) \times$ $I(k-1)$. For this function the result follows from Fact 114.

Lemma 7. For $k \geq 3$ and each $i=1,2, \ldots, 5 k-13$ the following inclusion holds:

$$
\left(\bigotimes \hat{Q}_{i \bmod 3+1}^{k}\right)\left(I\left(X_{i}^{k}\right)\right) \subseteq I\left(X_{i+1}^{k}\right) .
$$

Proof. We have to prove that for $k \geq 3$ and $x=1,2,3$ the following inclusions are true: $\left(\otimes \hat{Q}_{x}^{k}\right)\left(I\left(X_{3 j+x-1}^{k}\right)\right) \subseteq I\left(X_{3 j+x}^{k}\right)$, where $j=1,2, \ldots\left\lfloor\frac{5 k-13}{3}\right\rfloor$ for $x=1$ and $j=$ $0,1,2, \ldots\left\lfloor\frac{5 k-12-x}{3}\right\rfloor$ for $x=2,3$. The sequences $\hat{Q}_{x}^{k}, x=1,2,3$, are built of functions Cyc, Dec $*$, MinMax and Min introduced in Definition 7 We consider these function one after another analysing which positions in state sequences are modified by them and what values are in that positions before and after applying a function. In the following, we denote by $A_{i, j}$ the $j$-th element of a sequence $A_{i}$.

The function $C y c$ is used only in the definition of $\hat{Q}_{1}^{k}$ and is applied to position 1 of state sequences $I\left(X_{3 j}^{k}\right)$, where $j=1,2, \ldots\left\lfloor\frac{5 k-13}{3}\right\rfloor$. Thus it is enough to show the inclusion $\operatorname{Cyc}\left(I\left(X_{3 j, 1}^{k}\right)\right) \subseteq I\left(X_{3 j+1,1}^{k}\right)$. By Definition 11 the argument of $C y c \cdot I$ can be: $X_{3 j, 1}^{k}=V_{0,1}^{k}=-k$ for $3 j \leq 3 k-9$ or $X_{3 j, 1}^{k}=W_{0,1}^{k}=0$ for $3 j=3 k-6$ or $X_{3 j, 1}^{k}=Z_{1}=0$ for $3 j>3 k-6$. The corresponding value of the next state sequence is $X_{3 j+1,1}^{k}=V_{1,1}^{k}=k-1$ for $3 j+1 \leq 3 k-8$ or $X_{3 j+1,1}^{k}=Z_{1}=0$ for $3 j+1 \geq 3 k-5$. Using Fact 11 inclusions $C y c(I(-k)) \subseteq I(k-1)$ and $C y c(I(0)) \subseteq I(0)$ are true and we are done.

In the sequence $\hat{Q}_{1}^{k}$ we have several $D e c_{k-3 l}$ functions, each $D e c_{k-3 l}$ is on the corresponding position $3 l-1$ and it is applied to the state sequence $I\left(X_{3 j}^{k}\right)$, where $l=1, \ldots,\left\lfloor\frac{k-1}{3}\right\rfloor$. Similarly, in $\hat{Q}_{2}^{k}$ we have several $D e c_{k-3 l+1}$ functions, each $D e c_{k-3 l+1}$ is on the corresponding position $3 l-2$ and it is applied to the state sequence $I\left(X_{3 j+1}^{k}\right)$, where $l=1, \ldots,\left\lfloor\frac{k}{3}\right\rfloor$. Finally, in $\hat{Q}_{3}^{k}$ we have $D e c_{k-3 l-1}$ functions, each $D e c_{k-3 l-1}$ is on the corresponding position $3 l$ and it is applied to the state sequence $I\left(X_{3 j+2}^{k}\right)$, where $l=1, \ldots,\left\lfloor\frac{k-2}{3}\right\rfloor$. Assuming that $\hat{Q}_{0}^{k}$ also denotes $\hat{Q}_{3}^{k}$, we can rewrite our proof goal for that functions as the following fact.

Fact 14 For $k \geq 3$ and $x=0,1,2$ the set $\operatorname{Dec}_{k-3 l+x-1}\left(I\left(X_{3 j+x-1,3 l-x}^{k}\right)\right)$ is a subset of $I\left(X_{3 j+x, 3 l-x}^{k}\right)$, where $l=1, \ldots,\left\lfloor\frac{k-2+x}{3}\right\rfloor, j=1,2, \ldots\left\lfloor\frac{5 k-12-x}{3}\right\rfloor$ for $x=0,1$ and $j=$ $0,1,2, \ldots\left\lfloor\frac{5 k-14}{3}\right\rfloor$ for $x=2$.

The sequences $X_{*}^{k}$ are defined with the help of sequences $U_{*}^{k}, V_{*}^{k}, W_{*}^{k}$ and $Z_{*}$, therefore we prove the fact by considering all possible cases in the following table. In it we assume that $U_{-1}^{k}=U_{2}^{k}, V_{-1}^{k}=V_{2}^{k}$ and $W_{-1}^{k}=W_{2}^{k}$. 


\begin{tabular}{||l|l||c|c||c||}
\hline \hline \multicolumn{1}{|c|}{$\begin{array}{c}\text { Cases of } \\
s=X_{3 j+x-1,3 l-x}^{k}\end{array}$} & \multicolumn{1}{c|}{ Cases of } & Value of & Value of & Why \\
$3, j+x, 3 l-x$ & & $s$ & $t$ & $\operatorname{Dec}_{k-3 l+x-1}(I(s)) \subseteq I(t) ?$ \\
\hline \hline$s=U_{x-1,3 l-x}^{k}$ & $t=U_{x, 3 l-x}^{k}$ & $\pm k$ & $\pm k$ & \\
\hline$s=V_{x-1,3 l-x}^{k}$ & $t=V_{x, 3 l-x}^{k}$ & $k-3 l+x$ & $k-3 l+x-1$ & \multirow{2}{*}{ Fact 111} \\
\hline$s=V_{x-1,3 l-x}^{k}$ & $t=W_{x, 3 l-x}^{k}$ & $k-3 l+x$ & $k-3 l+x-1$ & \\
\hline$s=W_{x-1,3 l-x}^{k}$ & $t=W_{x, 3 l-x}^{k}$ & $k-3 l+x$ & $k-3 l+x-1$ & \\
\hline$s=Z_{3 l-x}$ & $t=Z_{3 l-x}$ & 0 & 0 & \\
\hline \hline
\end{tabular}

The two remaining cases: (1) $X_{3 j+x-1,3 l-x}^{k}=U_{x-1,3 l-x}^{k}$ and $X_{3 j+x, 3 l-x}^{k}=V_{x, 3 l-x}^{k}$ and (2) $X_{3 j+x-1,3 l-x}^{k}=W_{x-1,3 l-x}^{k}$ and $X_{3 j+x, 3 l-x}^{k}=Z_{3 l-x}$ are not possible, because, otherwise, (1) $3 j+x$ should be even and $\frac{3 j+x}{2}=3 l-x-1$, which cannot hold for any integers $j$, $x$ and $l$; (2) $3 j+x-(3 k-6)$ should be even and $\frac{3 j+x-(3 k-6)}{2}=3 l-x-1$, which is not true for the same reason.

Now we consider the Min function. It appears in the definition of $\hat{Q}_{1}^{k}\left(\hat{Q}_{2}^{k}\right.$ or $\hat{Q}_{3}^{k}$, respectively) on the position $k-2$ if $k \bmod 3=2(k \bmod 3=1$ or $k \bmod 3=0$, respectively). Thus, to prove the lemma, it suffices to show the following fact.

Fact 15 For $k \geq 3$ and $x=0,1,2$ the set $\operatorname{Min}\left(I\left(X_{3 j+x-1, k-2}^{k}\right)\right)$ is a subset of $I\left(X_{3 j+x, k-2}^{k}\right)$, where $k-2 \equiv 1-x(\bmod 3) j=1,2, \ldots\left\lfloor\frac{5 k-12-x}{3}\right\rfloor$ for $x=0,1$ and $j=0,1,2, \ldots\left\lfloor\frac{5 k-14}{3}\right\rfloor$ for $x=2$.

As in the case of $D e c_{*}$ functions we prove the fact by considering all possible cases in the following table. In it we assume that $U_{-1}^{k}=U_{2}^{k}, V_{-1}^{k}=V_{2}^{k}$ and $W_{-1}^{k}=W_{2}^{k}$.

\begin{tabular}{|c|c|c|c|c|}
\hline $\begin{array}{c}\text { Cases of } \\
s=X_{3 j+x-1, k-2}^{k}\end{array}$ & $\begin{array}{c}\text { Cases of } \\
t=X_{3 j+x, k-2}^{k} \\
\end{array}$ & $\begin{array}{c}\text { Value of } \\
s \\
\end{array}$ & $\begin{array}{c}\text { Value of } \\
t\end{array}$ & $\begin{array}{c}\text { Why } \\
\operatorname{Min}(I(s)) \subseteq I(t) ?\end{array}$ \\
\hline \multirow{2}{*}{$s=U_{x-1, k-2}^{k}$} & $t=U_{x, k-2}^{k}$ & $\pm k$ & $-k$ & \multirow{6}{*}{ Fact 113} \\
\hline & $t=V_{x, k-2}^{k}$ & $\pm k$ & $-k$ & \\
\hline$s=V_{x-1, k-2}^{k}$ & $t=W_{x, k-2}^{k}$ & 1 & 0 & \\
\hline \multirow{2}{*}{$s=W_{x-1, k-2}^{k}$} & $t=W_{x, k-2}^{k}$ & 1 & 0 & \\
\hline & $t=Z_{x, k-2}$ & 1 & 0 & \\
\hline$s=Z_{k-2}$ & $t=Z_{k-2}$ & 0 & 0 & \\
\hline
\end{tabular}

The remaining case $X_{3 j+x-1, k-2}^{k}=V_{x-1, k-2}^{k}$ and $X_{3 j+x, k-2}^{k}=V_{x, k-2}^{k}$ is not possible, because, otherwise $3 j+x-1=2 k-5$, that is, $2(k-2)=3 j+x$, but $k-2 \equiv 1-x(\bmod 3)$ and in the consequence $x \equiv 2(1-x)(\bmod 3)$ - contradiction.

The last function we have to consider is MinMax, which appears in the definition of all $\hat{Q}_{x}^{k}, x=1,2,3$, functions. In $\hat{Q}_{1}^{k}\left(\hat{Q}_{2}^{k}\right.$ and $\hat{Q}_{3}^{k}$, respectively) a copy of MinMax is on positions $(3,4),(6,7), \ldots((2,3),(5,6), \ldots$ and $(1,2),(4,5), \ldots$, respectively). Thus, to prove the lemma, it suffices to show the following fact.

Fact 16 For $k \geq 3$ and $x=1,2,3$ the set MinMax $\left(I\left(X_{3 j+x-1,3 l-x+1}^{k}, X_{3 j+x-1,3 l-x+2}^{k}\right)\right)$ is a subset of $I\left(X_{3 j+x, 3 l-x+1}^{k}, X_{3 j+x, 3 l-x+2}^{k}\right)$, where $l=1, \ldots,\left\lfloor\frac{k-4+x}{3}\right\rfloor j=1,2, \ldots\left\lfloor\frac{5 k-13}{3}\right\rfloor$ for $x=1$ and $j=0,1,2, \ldots\left\lfloor\frac{5 k-12-x}{3}\right\rfloor$ for $x=2,3$. 
As in the case of previous functions we prove the fact by considering all possible cases in the following table. In it we assume that $U_{3}^{k}=U_{0}^{k}, V_{3}^{k}=V_{0}^{k}$ and $W_{3}^{k}=W_{0}^{k}$. To reduce the size of the table we also use the following shortcuts: $a=3 j+x, b=3 l-x+1$ and $y=k-3 l+x-2$. Observe that $2 \leq y \leq k-2$, therefore $I(0) \subseteq I(y) \subseteq I( \pm k)$ and we can also apply Fact 115.

\begin{tabular}{|c|c|c|c|c|c|c|c|c|}
\hline \multicolumn{2}{|c|}{$\begin{array}{l}\text { Cases of }\left(s_{1}, s_{2}\right) \\
X_{a-1}^{k} \mid X_{a-1 h}^{k}\end{array}$} & $\begin{array}{l}\text { Cases c } \\
X_{a, b-1}^{k} \\
\end{array}$ & $\begin{array}{l}\mathrm{f}\left(t_{1}, t_{2}\right) \\
X_{a, b}^{k}\end{array}$ & Value of & $\begin{array}{c}\text { e of } \\
s_{2}\end{array}$ & $\begin{array}{l}\text { Val } \\
t_{1} \\
\end{array}$ & $\begin{array}{c}\mathrm{e} \text { of } \\
t_{2}\end{array}$ & $\begin{array}{c}\text { Why } I\left(t_{1}, t_{2}\right) \supseteq \\
\operatorname{MinMax}\left(I\left(s_{1}, s_{2}\right)\right) ?\end{array}$ \\
\hline \multirow{2}{*}{$U_{x-1, b-1}^{k}$} & \multirow{2}{*}{$U_{x-1, b}^{k}$} & $\overline{U_{x, b-1}^{k}}$ & $\overline{U_{x, b}^{k}}$ & $\overline{\bar{c} \pm k}$ & $-k$ & $-k$ & $\pm k$ & \multirow{2}{*}{ Fact 11,4} \\
\hline & & \begin{tabular}{|l|}
$V_{x, b-1}^{k}$ \\
\end{tabular} & $U_{x, b}^{k}$ & $\pm k$ & $-k$ & $-k$ & $\pm k$ & \\
\hline \multirow{5}{*}{$V_{x-1, b-1}^{k}$} & \multirow{2}{*}{$U_{x-1, b}^{k}$} & \multirow{2}{*}{$V_{x, b-1}^{k}$} & $U_{x, b}^{k}$ & $y$ & $-k$ & $-k$ & $\pm k$ & \multirow{10}{*}{ Fact 11.5} \\
\hline & & & $V_{x, b}^{k}$ & $y$ & $-k$ & $-k$ & $y$ & \\
\hline & \multirow{2}{*}{$V_{x-1, b}^{k}$} & \multirow{2}{*}{$V_{x, b-1}^{k}$} & $V_{x, b}^{k}$ & $y$ & $-k$ & $-k$ & $y$ & \\
\hline & & & $W_{x, b}^{k}$ & $y$ & $-k$ & $-k$ & $y$ & \\
\hline & $W_{x-1, b}^{k}$ & $W_{x, b-1}^{k}$ & $W_{x, b}^{k}$ & $y$ & 0 & 0 & $y$ & \\
\hline \multirow{2}{*}{$W_{x-1, b-1}^{k}$} & \multirow{2}{*}{$W_{x-1, b}^{k}$} & $W_{x, b-1}^{k}$ & $W_{x, b}^{k}$ & $y$ & 0 & 0 & $y$ & \\
\hline & & $Z_{b-1}$ & $W_{x, b}^{k}$ & $y$ & 0 & 0 & $y$ & \\
\hline \multirow{3}{*}{$Z_{b-1}$} & \multirow{2}{*}{$W_{x-1, b}^{k}$} & \multirow{2}{*}{$Z_{b-1}$} & $W_{x, b}^{k}$ & 0 & 0 & 0 & $y$ & \\
\hline & & & $Z_{b}$ & 0 & 0 & 0 & 0 & \\
\hline & $Z_{b}$ & $Z_{b-1}$ & $Z_{b}$ & 0 & 0 & 0 & 0 & \\
\hline
\end{tabular}

Lemma 8. Let $k \geq 3$ and let $\bar{c}=\left(c_{1}, \ldots, c_{b_{k}}\right)$ be a balanced 2-flat sequence of integers from $\left[0,2^{k-1}-1\right]$ and let $s=$ height $(\bar{c})$. Let $f=f_{5 k-12} \circ f_{5 k-13} \circ \ldots \circ f_{1}$, where $f_{i}=$ $Q_{((i-1) \text { mod } 3)+1}^{k}, i=1, \ldots, 5 k-12$. Then $f(\bar{c})=\left(\frac{s}{2}\right)^{b_{k}}$ if s is even or $f(\bar{c})=\left(\frac{s-1}{2}\right)^{k-2} \oplus$ $\left(\frac{s+1}{2}\right)^{k-2}$ otherwise.

Proof. Since each $f_{i}$ maps a balanced sequence to a balanced one, let $\hat{f}_{i}=\operatorname{reduce}\left(f_{i}, s\right)=$ $\otimes \hat{Q}_{((i-1) \bmod 3)+1}^{k}$, where the later equality follows from Lemma 5. Let also $\bar{d}_{0}=$ reduce $(\bar{c})$ and let $\bar{d}_{i}=\hat{f}_{i}\left(\bar{d}_{i-1}\right)$ for $i=1, \ldots, 5 k-12$. Then $\bar{d}_{1} \in I\left(X_{1}^{k}\right)$ by Lemma 6 and for $i=2, \ldots, 5 k-12$ we get $\bar{d}_{i} \in I\left(X_{i}^{k}\right)$ by an easy induction and Lemma7 7 . Let $\mathbb{Z}$ denote as usual the set of integers. By $\mathbb{Z}_{\frac{1}{2}}$ we will denote the set $\left\{z+\frac{1}{2} \mid z \in \mathbb{Z}\right\}$. Looking at Definitions 6 and 7 observe the following fact:

Fact 17 If $s$ is even then all elements of sequences $\bar{d}_{i}, i=0, \ldots, 5 k-12$, are integers. If $s$ is odd then all elements of sequences $\bar{d}_{i}, i=0, \ldots, 5 k-12$, are in $\mathbb{Z}_{\frac{1}{2}}$.

Since $\bar{d}_{5 k-12} \in I\left(X_{5 k-12}^{k}\right)=I\left(0^{k-2}\right)$ and $I(0) \cap \mathbb{Z}=\{0\}$ and $I(0) \cap \mathbb{Z}_{\frac{1}{2}}=\left\{\frac{1}{2}\right\}$, it follows that $\bar{d}_{5 k-12}=0^{k-2}$ if $s$ is even and $\bar{d}_{5 k-12}=\frac{1}{2}^{k-2}$, otherwise. Applying now the definition of $s$-extended sequence to $0^{k-2}$ and $\frac{1}{2}^{k-2}$ we get the desired conclusion of the lemma.

In this way, with respect to Lemma 3 , we have proved that the network $M_{k}$ is able to merge in $5 k-12$ stages two sorted sequences given in odd and even registers, provided that the numbers of ones in our matrix columns form a balanced sequence. If the sequence is not balanced, $k-3$ additional stages are needed to get a sorted output. 


\subsection{Analysis of General Columns}

In a general case we will use balanced sequences as lower and upper bounds on the numbers of ones in our matrix columns and observe that $Q_{1}^{k}, Q_{2}^{k}$ and $Q_{3}^{k}$ are monotone functions (see Fact 9).

Definition 12. Let $k \geq 3$ and let $\bar{c}=\left(c_{1}, \ldots, c_{b_{k}}\right)$ be a 2-flat sequence of integers from $\left[0,2^{k-1}-1\right]$ that is not balanced. Since both $\bar{c}_{\text {odd }}=\left(c_{1}, \ldots, c_{b_{k}-1}\right)$ and $\bar{c}_{\text {evn }}=\left(c_{2}, \ldots, c_{b_{k}}\right)$ are flat sequences, let $i$ ( $j$, respectively) be such that $c_{2 i-1}<c_{2 i+1}\left(c_{b_{k}-2 j}<c_{b_{k}-2 j+2}\right.$, respectively) or let $i=k-2(j=k-2)$ if $\bar{c}_{\text {odd }}\left(\bar{c}_{\text {evn }}\right.$, respectively) is a constant sequence. The defined below sequences $\check{c}$ and $\hat{c}$ we will call lower and upper bounds of $\bar{c}$. If $i<j$ then for $l=1, \ldots, b_{k}$

$$
\check{c}_{l}=\left\{\begin{array}{ll}
c_{1} & \text { if } l \text { is odd and } l \leq 2 j-1 \\
c_{b_{k}-1} & \text { if } l \text { is odd and } l \geq 2 j+1 \\
c_{l} & \text { if } l \text { is even }
\end{array} \quad \hat{c}_{l}= \begin{cases}c_{b_{k}-1} \text { if } l \text { is odd } \\
c_{b_{k}} \text { if } l \text { is even }\end{cases}\right.
$$

If $i>j$ then for $l=1, \ldots, b_{k}$

$$
\check{c}_{l}=\left\{\begin{array}{l}
c_{1} \text { if } l \text { is odd } \\
c_{2} \text { if } l \text { is even }
\end{array} \quad \hat{c}_{l}= \begin{cases}c_{l} \text { if } l \text { is odd } \\
c_{2} \text { if } l \text { is even and } l \leq b_{k}-2 i \\
c_{b_{k}} \text { if } l \text { is even and } l>b_{k}-2 i\end{cases}\right.
$$

Fact 18 For $k \geq 3$ and any not balanced 2 -flat sequence $\bar{c}=\left(c_{1}, \ldots, c_{b_{k}}\right)$ of integers from $\left[0,2^{k-1}-1\right]$ the sequences $\check{c}$ and $\hat{c}$ are balanced, height $(\check{c})+1=$ height $(\hat{c})$ and $\check{c} \leq \bar{c} \leq \hat{c}$.

Proof. Let $i$ and $j$ be defined as in Definition 12 We will only consider the case $i<j$. The proof of the other case is similar. Directly from the definition we get that $\hat{c}$ is balanced. To see that $\check{c}$ is also balanced let us check for $l=1, \ldots, k-2$ whether the sum $\check{c}_{2 l-1}+\check{c}_{b_{k}-2 l+2}$ is constant.

$$
\check{c}_{2 l-1}+\check{c}_{b_{k}-2 l+2}=\check{c}_{2 l-1}+c_{b_{k}-2 l+2}= \begin{cases}c_{1}+c_{b_{k}-2 l+2}=c_{1}+c_{b_{k}} & \text { if } l \leq j \\ c_{b_{k}-1}+c_{b_{k}-2 l+2}=c_{b_{k}-1}+c_{2} & \text { otherwise }\end{cases}
$$

If $j=k-2$ there is no otherwise case and we are done. If $j<k-2$ then $c_{b_{k}}-c_{2}=$ $c_{b_{k}-1}-c_{1}=1$, because of the definition of $i$ and $j$ and we are also done. Moreover height $(\check{c})+1=c_{1}+c_{b_{k}}+1=c_{b_{k}-1}+c_{b_{k}}=\operatorname{height}(\hat{c})$. To prove that $\check{c} \leq \bar{c} \leq \hat{c}$ we consider even and odd indices. For even indices from the definition we have: $\check{c}_{2 l}=c_{2 l} \leq$ $c_{b_{k}}=\hat{c}_{2 l}$. For odd indices $\hat{c}_{2 l-1}=c_{b_{k}-1} \geq c_{2 l-1} \geq c_{1}$. If $l \leq j$ we are done, otherwise, $c_{2 l-1}=c_{b_{k}-1}=\check{c}_{2 l-1}$, because $\bar{c}_{\text {odd }}$ is flat.

Theorem 19. Let $k \geq 3$ and let $\bar{c}=\left(c_{1}, \ldots, c_{b_{k}}\right)$ be a 2-flat sequence of integers from $\left[0,2^{k-1}-1\right]$. Let $f=f_{6 k-15} \circ f_{6 k-14} \circ \ldots \circ f_{1}$, where $f_{i}=Q_{((i-1) \text { mod } 3)+1}^{k}, i=1, \ldots, 6 k-$ 15. Then $f(\bar{c})$ is a flat sequence.

Proof. For a a 2-flat sequence $\bar{c}$ of integers from $\left[0,2^{k-1}-1\right]$ let $\check{c}$ and $\hat{c}$ be its balanced lower and upper bounds, as defined in Definition 12 Let $\bar{c}_{0}=\bar{c}, \check{c}_{0}=\check{c}, \hat{c}_{0}=\hat{c}$ and for $i=1, \ldots, 6 k-15$ let us define $\bar{c}_{i}=f_{i}\left(\bar{c}_{i-1}\right), \check{c}_{i}=f_{i}\left(\check{c}_{i-1}\right)$ and $\hat{c}_{i}=f_{i}\left(\hat{c}_{i-1}\right)$. Observe that $\check{c}_{i} \leq \bar{c}_{i} \leq \hat{c}_{i}$, because of monotonicity of functions $Q_{1}^{k}, Q_{2}^{k}, Q_{3}^{k}$ and Fact 18 To prove that $\bar{c}_{6 k-15}$ is a flat sequence we need the following three technical facts. 
Fact 20 Let $s=$ height $(\check{c})$. If $s$ is even then $\bar{c}_{i, j}=\frac{s}{2}$ and $\bar{c}_{i, b_{k}-j+1} \in\left\{\frac{s}{2}, \frac{s}{2}+1\right\}$ for each $i=3 k-6, \ldots, 5 k-12$ and $j=1, \ldots,\left\lceil\frac{i+1-(3 k-6)}{2}\right\rceil$. If s is odd then $\bar{c}_{i, j} \in\left\{\frac{s-1}{2}, \frac{s+1}{2}\right\}$ and $\bar{c}_{i, b_{k}-j+1}=\frac{s+1}{2}$ for each $i=3 k-6, \ldots, 5 k-12$ and $j=1, \ldots,\left\lceil\frac{i+1-(3 k-6)}{2}\right\rceil$.

Proof. Since both $\check{c}$ and $\hat{c}$ are balanced, we can consider reduced forms of them and use Lemmas 6 and 7 For the given range of $i$ 's values that means that

$$
\operatorname{reduce}\left(\check{c}_{i}\right), \operatorname{reduce}\left(\hat{c}_{i}\right) \in I\left(X_{i}^{k}\right)=I\left(\operatorname{join}_{k}\left(\left\lceil\frac{i+1-(3 k-6)}{2}\right\rceil, Z^{k}, W_{i}^{k}\right)\right) .
$$

It follows that for a given range of $j$ 's values reduce $\left(\check{c}_{i}\right)_{j}$, reduce $\left(\hat{c}_{i}\right)_{j} \in I(0)=\left[-\frac{1}{2}, 0\right]$. From Fact 18 we know that height $(\hat{c})=s+1$ and from Lemma 4 that heights are preserved in sequences $\check{c}_{i}$ and $\hat{c}_{i}$. Thus, from the definition of a reduced sequence, $\check{c}_{i, j} \in$ $\left[\frac{s-1}{2}, \frac{s}{2}\right], \check{c}_{i, b_{k}-j+1} \in\left[\frac{s}{2}, \frac{s+1}{2}\right], \hat{c}_{i, j} \in\left[\frac{s}{2}, \frac{s+1}{2}\right]$ and $\hat{c}_{i, b_{k}-j+1} \in\left[\frac{s+1}{2}, \frac{s+2}{2}\right]$. Since $\check{c}_{i}$ and $\hat{c}_{i}$ are sequences of integers, for even $s$ we get $\check{c}_{i, j}=\check{c}_{i, b_{k}-j+1}=\hat{c}_{i, j}=\frac{s}{2}$ and $\hat{c}_{i, b_{k}-j+1}=\frac{s+2}{2}$; for odd $s$ we conclude that $\check{c}_{i, j}=\frac{s-1}{2}$ and $\check{c}_{i, b_{k}-j+1}=\hat{c}_{i, j}=\hat{c}_{i, b_{k}-j+1}=\frac{s+1}{2}$. Since $\check{c}_{i, j} \leq \bar{c}_{i, j} \leq \hat{c}_{i, j}$, the fact follows.

The second fact extends the first fact up to the last stage of our computation.

Fact 21 Let $s=$ height $(\check{c})$. If $s$ is even then $\bar{c}_{i, j}=\frac{s}{2}$ and $\bar{c}_{i, b_{k}-j+1} \in\left\{\frac{s}{2}, \frac{s}{2}+1\right\}$ for each $i=5 k-11, \ldots, 6 k-15$ and $j=1, \ldots, k-2$. If $s$ is odd then $\bar{c}_{i, j} \in\left\{\frac{s-1}{2}, \frac{s+1}{2}\right\}$ and $\bar{c}_{i, b_{k}-j+1}=\frac{s+1}{2}$ for each $i=5 k-11, \ldots, 6 k-15$ and $j=1, \ldots, k-2$.

Proof. Consider first the sequence $\bar{c}_{5 k-12}$ and observe that for $i=5 k-12$ the value of $\left\lceil\frac{i+1-(3 k-6)}{2}\right\rceil$ is equal to $k-2$. It follows from Fact 20 that for even $s$ all values from the left half of $\bar{c}_{5 k-12}$ are equal to $\frac{s}{2}$ and all values from the right half of $\bar{c}_{5 k-12}$ are in $\left\{\frac{s}{2}, \frac{s}{2}+1\right\}$. For odd $s$ all values from the left half of $\bar{c}_{5 k-12}$ are in $\left\{\frac{s-1}{2}, \frac{s+1}{2}\right\}$ and all values from the right half of $\bar{c}_{5 k-12}$ are equal to $\frac{s+1}{2}$. Since $Q_{1}^{k}, Q_{2}^{k}$ and $Q_{3}^{k}$ are built of functions $d e c_{*}^{k}, m o v_{*}^{k}$ and $c y c^{k}$ (cf. Definitions 3 and 4) observe that each function $f_{i}$, $i=5 k-11, \ldots, 6 k-15$ can only exchange values at positions from $\operatorname{args}\left(\operatorname{mov}_{*}^{k}\right)$ that are from non-constant half of arguments (in case of $d e c_{*}^{k}$ and $c y c^{k}$ we can observe that for $a \leq b \leq a+1$ and any $h \geq 0$ we have $\min (a, b+h)=a, \max (a-h, b)=b$, $\max (a, b-1)=a$ and $\min (a+1, b)=b$, that is, the functions are identity mappings in stages $5 k-11, \ldots, 6 k-15)$. The $m o v_{*}^{k}$ functions can only exchange unequal values at neighbour positions moving the smaller value to the left.

The last fact states that unequal values $\bar{c}_{i, j}$ described in the previous two facts are getting sorted during the computation. Observe that if $s$ is odd (even, respectively) then we only have to trace the sorting process in a left (right, respectively) region of indices $\left[1, \min \left(k-2,\left\lceil\frac{i+1-(3 k-6)}{2}\right\rceil\right)\right]\left(\left[\max \left(k-1, b_{k}-\left\lceil\frac{i+1-(3 k-6)}{2}\right\rceil+1\right), b_{k}\right]\right.$, respectively), where $i=3 k-6, \ldots, 6 k-15$ and the values to be sorted differs at most by one. We trace the positions of the smaller values $s^{\prime}=\frac{s-1}{2}$ in the left region and the greater values $s^{\prime}=\frac{s}{2}+1$ in the right region. We will call $s^{\prime}$ a moving element. For $t=1, \ldots, k-2$ let us define $i_{t}=3 k+2 t-8$ to be the stage, after which the length of the region extends from $t-1$ to $t$ and a new element appears in it. Let $t^{\prime}=t$ for odd $s$ and $t^{\prime}=b_{k}-t+1$, 
otherwise, be the position of this new element and $a_{t}=c_{i_{t}, t^{\prime}}$ be its value. Finally, let $n_{t}=\left|\left\{1 \leq l \leq t \mid a_{l}=s^{\prime}\right\}\right|$ be the number of moving elements in the region after stage $i_{t}$.

Fact 22 Using the above definitions, for $t=1, \ldots, k-2$, if $a_{t}=s^{\prime}$ then for $i=0, \ldots, 6 k-$ $15-i_{t}$ we have $c_{i_{t}+i, \max \left(t-i, n_{t}\right)}=a_{t}$ if $s$ is odd and $c_{i_{t}+i, \min \left(t^{\prime}+i, b_{k}-n_{k}+1\right)}=a_{t}$, otherwise.

Proof. We prove the fact only for odd $s$, that is, for the left region. The proof for the right region is symmetric. We would like to show that if $a_{t}=s^{\prime}$ appears at position $t^{\prime}=t$ after stage $i_{t}$ then it moves in each of the following stages one position to the left up to its final position $n_{t}$. The proof is by induction on $t$ and $i$. If $t=1$ and $a_{1}=s^{\prime}$ appears at position 1 after stage $i_{1}=3 k-6$ then $n_{1}=1$ and $a_{1}$ is already at its final position. It never moves, because values at second position are $\geq s^{\prime}$, by Facts 20 and 21 If $t>1$ and $a_{t}=s^{\prime}$ then the basis $i=0$ is obviously true. In the inductive step $i>0$ we assume that $c_{i_{t}+i-1, \max \left(t^{\prime}-i+1, n_{t}\right)}=a_{t}$ and that the fact is true for smaller values of $t$. If $\max \left(t-i+1, n_{t}\right)=n_{t}$ then also $\max \left(t-i, n_{t}\right)=n_{t}$ and, by the induction hypothesis, values at positions $1, \ldots, n_{t}-1$ are all equal $s^{\prime}$. That means that $a_{t}$ is at its final position and we are done. Thus we left with the case: $n_{t}<t-i+1$, that is, with $n_{t} \leq t-i$.

Consider the sequences $\bar{c}_{i_{t}+i-1}$ and $\bar{c}_{i_{t}+i}=f_{i_{t}+i}\left(\bar{c}_{i_{t}+i-1}\right)$. We know that $\bar{c}_{i_{t}+i-1, t-i+1}=$ $s^{\prime}$. To prove that $\bar{c}_{i_{t}+i, t-i}=s^{\prime}$ we would like to show that $\bar{c}_{i_{t}+i-1, t-i}=s^{\prime}+1$ and $m o v_{t-i}^{k} \in$ $f_{i_{t}+i}$. The later is a direct consequence of an observation that $m o v_{a}^{k} \in f_{b}$ if and only if $(a+b) \equiv 1(\bmod 3)$. In our case $(t-i)+\left(i_{t}+i\right)=t+i_{t}=t+3 k+2 t-8 \equiv 1(\bmod 3)$. To prove the former, let us consider $a_{u}=s^{\prime}, u \leq t-1$. Then $i_{u} \leq i_{t}-2$ and $n_{u} \leq n_{t}-1$. By the induction hypothesis, $c_{i_{u}+j, \max \left(u-j, n_{u}\right)}=s^{\prime}$. Setting $j=i_{t}-i_{u}+i-1$ we get $j \geq i+1$ and $\max \left(u-j, n_{u}\right) \leq \max \left(t-1-(i+1), n_{t}-1\right)<\max \left(t-i, n_{t}\right)=t-i$. Moreover, $i_{u}+j=i_{t}+i-1$. That means that in the sequence $\bar{c}_{i_{t}+i-1}$ none of $n_{t}$ elements $s^{\prime}$ is at position $t-i$ and, consequently, $\bar{c}_{i_{t}+i-1, t-i}=s^{\prime}+1$. Since $m o v_{t-i}^{k}$ switches $s^{\prime}$ with $s^{\prime}+1$, this completes the proof of Fact 22

Now we are ready to prove that $\bar{c}_{6 k-15}$ is a flat sequence. By Fact 21, if $s$ is odd then $\bar{c}_{6 k-15} \in\left\{\frac{s-1}{2}, \frac{s+1}{2}\right\}^{k-2}\left(\frac{s+1}{2}\right)^{k-2}$, otherwise, $\bar{c}_{6 k-15} \in\left(\frac{s}{2}\right)^{k-2}\left\{\frac{s}{2}, \frac{s}{2}+1\right\}^{k-2}$. The number of minority elements in $\bar{c}_{6 k-15}$ has been denote by $n_{k-2}$. If $s$ is odd and $a_{t}, t=1, \ldots, k-$ 2 , is a minority element $\frac{s-1}{2}$, then, by Fact 22, $c_{6 k-15, n_{t}}=\frac{s-1}{2}$. If $s$ is even and $a_{t}$, $t=1, \ldots, k-2$, is a minority element $\frac{s}{2}+1$, then, by Fact $22, c_{6 k-15, b_{k}-n_{t}+1}=\frac{s}{2}+2$. In both cases this proves that $\bar{c}_{6 k-15}$ is flat, which completes the proof of Theorem 19

\subsection{Proof of Theorem 2}

Theorem 2 follows directly from Theorem 19 and Lemma 3 . Let $k \geq 3$ and $\bar{c}$ be any 2 -flat sequence of integers from $\left[0,2^{k-1}-1\right]$. By Theorem 19 the result of application $\left(Q_{3}^{k} \circ Q_{2}^{k} \circ Q_{1}^{k}\right)^{2 k-5}$ to $(\bar{c})$ is a flat sequence. Then, by Lemma 3 , the network $M_{k}$ is a $2 k-$ 5 -pass merger of two sorted sequences given in odd and even registers, respectively.

\section{Conclusions}

For each $k \geq 3$ we have shown a construction of a 3-periodic merging comparator network of $N_{k}=2^{k}(k-2)$ registers and proved that it merge any two sorted sequences 
(given in odd and even registers, respectively) in time $6 k-15=3(k-5)$. A natural question remains whether it is the optimal merging time for 3-periodic comparator networks.

\section{References}

1. M. Ajtai, J. Komlos And E. Szemeredi, $A n O(n \log n)$ sorting network, in Proc. 15 th Annual ACM Symp. on Theory of Computing, 1983, pp. 1-9.

2. K.E. BATCHER, Sorting networks and their applications, in Proc. AFIPS 1968 SJCC, Vol. 32, AFIPS Press, Montvale, NJ, pp. 307-314.

3. E. A. Bender and S. G. WILliamson, Periodic Sorting Using Minimum Delay, Recursively Constructed Merging Networks, The Electronic Journal of Combinatorics 5 (1998), pp. 1-21.

4. E. R. CANFIELD AND S. G. Williamson, A sequential sorting network analogous to the Batcher merge, Linear and Multilinear Algebra, 29 (1991), pp. 43-51.

5. M. DOWD, Y. PERL, M. SAKS AND L. RUdOLPH, The periodic balanced sorting network, Journal of ACM, 36 (1989), pp. 738-757.

6. D.E. KNUTH, The Art of Computer Programming, Vol. 3, 2nd edition, Addison Wesley, Reading, MA, 1975.

7. M. KutyŁowski, K. Loryś And B. Oesterdiekhoff, Periodic Merging Networks, Theory of Computing Systems, 31.5 (1998), pp. 551-578.

8. M. KutyŁowski, K. Loryś, B. Oesterdiekhoff And R. Wanka, Periodification scheme: constructing sorting networks with constant period, Journal of ACM, 47 (2000), pp. 944-967.

9. F.T. Leighton, Introduction to Parallel Algorithms and Architectures: Arrays, Trees and Hypercubes, Morgan-Kaufmann, San Mateo, CA, 1992.

10. T. LeVi And A. Litman, The Strongest Model of Computation Obeying 0-1 Principles, Theory of Computing Systems, 48(2) (2011), pp. 374-388.

11. P.B. Miltersen, M. PAterson And J. TARUi, The asymptotic complexity of merging networks, Journal of the ACM, 43(1) (1996), pp. 147-165.

12. B. OesterdieKHOFF, Periodic comparator networks, Theoretical Computer Science, 245 (2000), pp. 175-202.

13. M. Piotrów, Periodic, Random-Fault-Tolerant Correction Networks, in Proc. 13th ACM Symposium on Parallel Algorithms and Architectures, ACM Press, New York, 2001.

14. M. Piotrów, A note on Constructing Binary Heaps with Periodic Networks, Information Processing Letters, 83 (2002), pp. 129-134.

15. J. SEIFERAS, Research note: Networks for sorting multitonic sequences, Journal on Parallel Distrib. Comput., 65(12) (2005), pp. 1601-1606. 


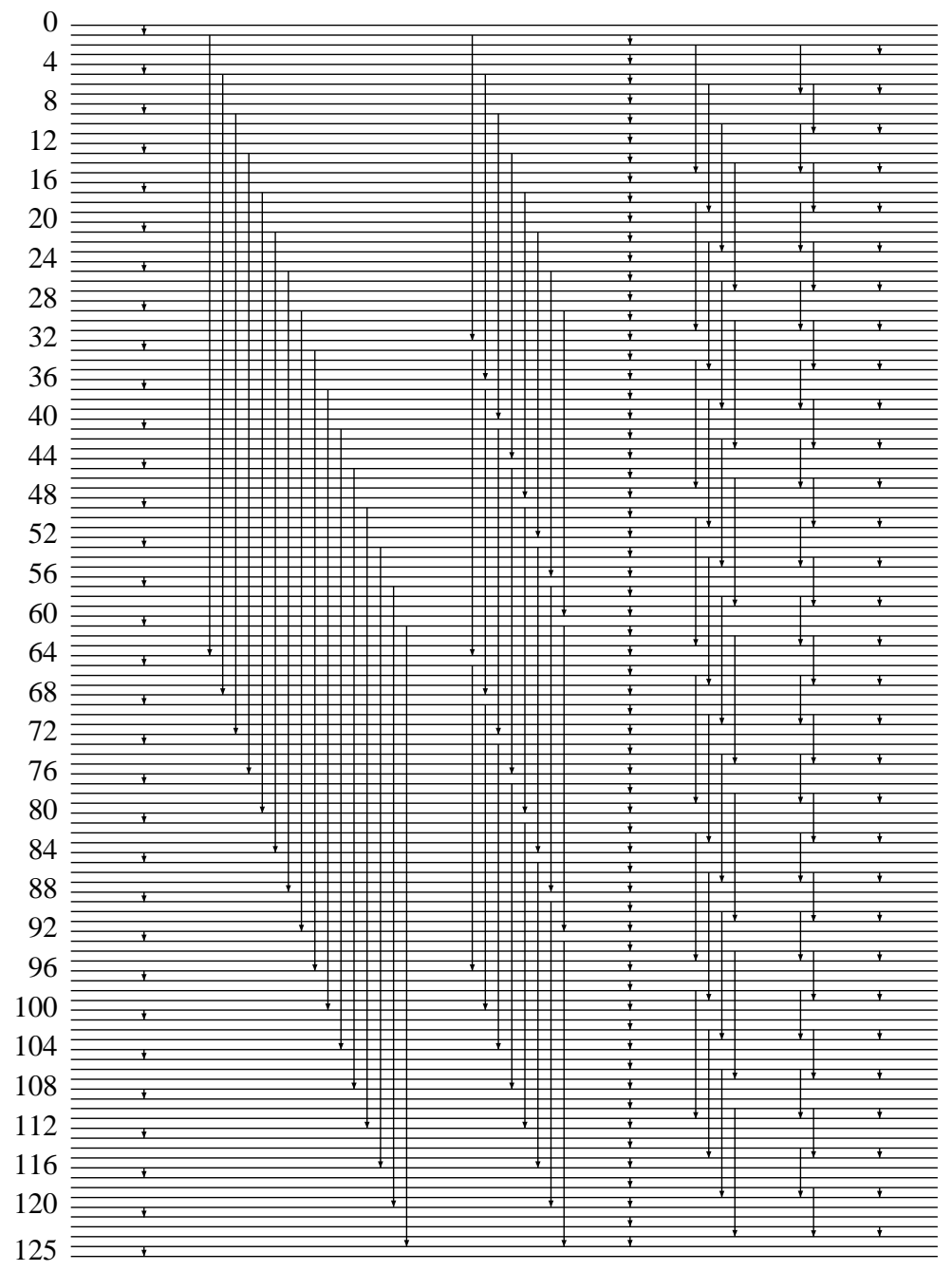

Fig. 6. The $P_{6}^{\prime}$ network 